\title{
Current State of Porous Carbon for Wastewater Treatment
}

\author{
Mongi ben Mosbah ${ }^{1,2}$, Lassaad Mechi ${ }^{1,3}$, Ramzi Khiari $4,5,6 \oplus$ and Younes Moussaoui ${ }^{2,7, *(1)}$ \\ 1 Materials, Environment and Energy Laboratory (UR14ES26), Faculty of Sciences of Gafsa, \\ University of Gafsa, Gafsa 2112, Tunisia; mbenmosbah@yahoo.fr (M.b.M.); mechilassaad@yahoo.fr (L.M.) \\ 2 Faculty of Sciences of Gafsa, University of Gafsa, Gafsa 2112, Tunisia \\ 3 Department of Chemistry, Faculty of Sciences, University of Hail, Hail PO Box 2440, Saudi Arabia \\ 4 Higher Institute of Technological Studies, Ksar Hellal 5070, Tunisia; khiari_ramzi2000@yahoo.fr \\ 5 UR13 ES 63-Research Unity of Applied Chemistry \& Environment, Faculty of Sciences, \\ University of Monastir, Monastir 5000, Tunisia \\ 6 LGP2, University of Grenoble Alpes, CNRS, Grenoble INP, LGP2, F-38000 Grenoble, France \\ 7 Organic Chemistry Laboratory (LR17ES08), Faculty of Sciences of Sfax, University of Sfax, Sfax 3029, Tunisia \\ * Correspondence: y.moussaoui2@gmx.fr
}

Received: 5 August 2020; Accepted: 8 December 2020; Published: 14 December 2020

\begin{abstract}
Porous materials constitute an attractive research field due to their high specific surfaces; high chemical stabilities; abundant pores; special electrical, optical, thermal, and mechanical properties; and their often higher reactivities. These materials are currently generating a great deal of enthusiasm, and they have been used in large and diverse applications, such as those relating to sensors and biosensors, catalysis and biocatalysis, separation and purification techniques, acoustic and electrical insulation, transport gas or charged species, drug delivery, and electrochemistry. Porous carbons are an important class of porous materials that have grown rapidly in recent years. They have the advantages of a tunable pore structure, good physical and chemical stability, a variable specific surface, and the possibility of easy functionalization. This gives them new properties and allows them to improve their performance for a given application. This review paper intends to understand how porous carbons involve the removal of pollutants from water, e.g., heavy metal ions, dyes, and organic or inorganic molecules. First, a general overview description of the different precursors and the manufacturing methods of porous carbons is illustrated. The second part is devoted to reporting some applications such using porous carbon materials as an adsorbent. It appears that the use of porous materials at different scales for these applications is very promising for wastewater treatment industries.
\end{abstract}

Keywords: porous materials; activated carbon; biomass; activating agent; adsorption; applications; pollutants

\section{Introduction}

Porous materials are defined as materials with cavities, or channels, called pores. Pores are empty spaces that exist between particles of any shape in materials. These empty spaces form the porosity of the materials, defined as the volume percentage of air in the material that correspond to the total volume occupied by the voids of the material divided by the total volume of the material [1]. The porous space is a continuity of absence of solid matter nested in the continuity of solid matter. It is essentially irregular in its forms and inconsistent also in its qualities, which can be given great complexity. There are two types of porosity: open porosity (on the external environment) and closed porosity. In all cases, it is generally characterized by its volume fraction (or by the density of the porous 
material) and the pore size distribution. The open porosity notably controls the adhesion properties of the coatings on the surface of the material [2].

Porous materials have attracted the attention of chemists and materials scientists, and the development of new porous materials has accelerated research development in recent decades [3-5]. This attention comes back not only to the commercial interest that porous materials have for their application in various fields such as separation [6,7], catalysis [7-9], adsorption [6,10-15], energy storage and conversion [16-24], and medicine [25,26], but also, because of the scientific interest in the challenges posed by their synthesis, their treatment and their characterization. Indeed, the progress of technology and the demand for porous materials have pushed researchers to develop synthesis methods allowing for controlling the parameters that determine the structural and textural characteristics of these materials. In this context, a lot of works have shown that controlling the pore size is essential for many specific applications. However, this technological and industrial development with the growth of the world population and domestic activities are causing a remarkable increase in water pollution. It is therefore necessary to purify wastewater before it is released into the environment. Thus, several wastewater treatment technologies have been developed, the adsorption of which on porous materials is proving to be one of the most promising techniques for the removal of pollutants from wastewater due to its affordable price and ease of disposal at large-scale use [27-30]. The most widely used adsorbents are activated carbons, and they are considered the most useful for the removal of organic and inorganic pollutants due to their structural variability (macro-, meso-, and micropores), their large specific surface area, and a wide availability of functional groups [29,31,32].

The purpose of this review is to discuss the preparation processes of porous carbons as well as the main carbon precursors used. In addition, the performance and characteristics of the resulting carbons are investigated. Finally, the contribution of activated carbon in the field of wastewater treatment will also be discussed in detail.

\section{Classification of Porous Materials}

According to the International Union of Pure and Applied Chemistry-USA(IUPAC) [33,34], porous materials are classified according to the diameter of the pores $\left(d_{\mathrm{p}}\right)$ that constitute them, and the three classes are seen in Figure 1.

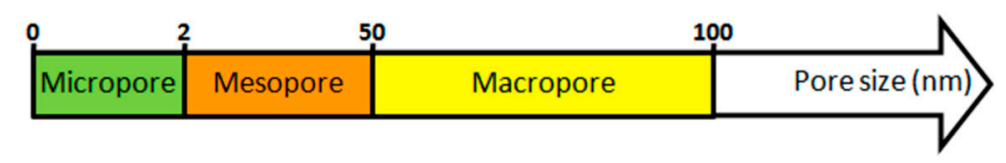

Figure 1. Classification of porosity based on pore diameter according to the International Union of Pure and Applied Chemistry (IUPAC).

\subsection{Microporous Materials}

Microporous materials are those whose pore diameter is less than $2 \mathrm{~nm}\left(d_{\mathrm{p}}<2 \mathrm{~nm}\right)$. They can be prepared by the sol-gel process and are widely used in catalysis and adsorption.

\subsection{Mesoporous Materials}

In this category of materials, the pore diameter varies between 2 and $50 \mathrm{~nm}\left(2 \mathrm{~nm}<d_{\mathrm{p}}<50 \mathrm{~nm}\right)$. A distinction is made between crystalline mesoporous materials and ordered amorphous materials, which are intermediate between that of crystalline microporous solids of the zeolite type and disordered amorphous solids such as silica gel [35-37]. Mesoporous materials have several known applications such as catalysis, filtration, pollution control, optics, and even electronics. 


\subsection{Macroporous Materials}

As these materials have fairly large pores $\left(d_{\mathrm{p}}>50 \mathrm{~nm}\right)$, they find their use in the field of decontamination of polluted water with organic dyes and as a catalyst support for the photodegradation of the pollutant $[20,38-41]$.

\section{Graphite and Graphene}

Graphite consists of interconnected carbon atoms arranged to hexagonally form flat networks in the form of layers stacked parallel to each other (Figure 2b) [42,43]. These layers are connected by low energy van der Waals type bonds. This explains the laminated morphology of graphite [42,43]. Graphite is characterized by high thermal and electrical conductivity, good chemical stability, and remarkable plasticity [43-50]. A good thermal and electrical conductivity is attributed to the delocalization of the $\pi$-electrons of the carbon atoms in graphite, and its plasticity can be explained by the possibility of sliding the sheets layers one onto the other [42,43]. These favorable characteristics make graphite interesting materials for improving the transfer and storage of thermal energy, for preparing anodes of lithium-ion batteries, and for electrochemical applications. In this context, Zhong et al. [51] used pitch-based graphite foams to increase the thermal diffusivity of paraffin wax for a thermal energy storage application. Later, Jana et al. [45] studied the effect of the addition of graphite on the conductivity of a tannin-based resin. They showed, contrary to what was expected a priori, that the smaller graphite particles were much more suitable for obtaining conductive matrices. Indeed, the use of small grains made the viscosity higher because of their higher surface and made it possible to obtain homogeneous foams with a higher density [45]. In 2019, Zhang et al. [52] showed that the use of graphitized mesoporous carbon as anode materials for lithium-ion batteries improved speed performance and had a good cycling stability with a reversible capacity of $248.3 \mathrm{~mA} \mathrm{~h} \mathrm{~g}^{-1}$ at $1 \mathrm{C}$ after 100 cycles. In the same context, porous graphitic carbon was synthesized from Eichhornia crassipes plants collected from the Coimbatore region, Tamilnadu-India, and has been used for the sustainable fabrication of hole-transporting materials electrode in perovskite solar cells [53].
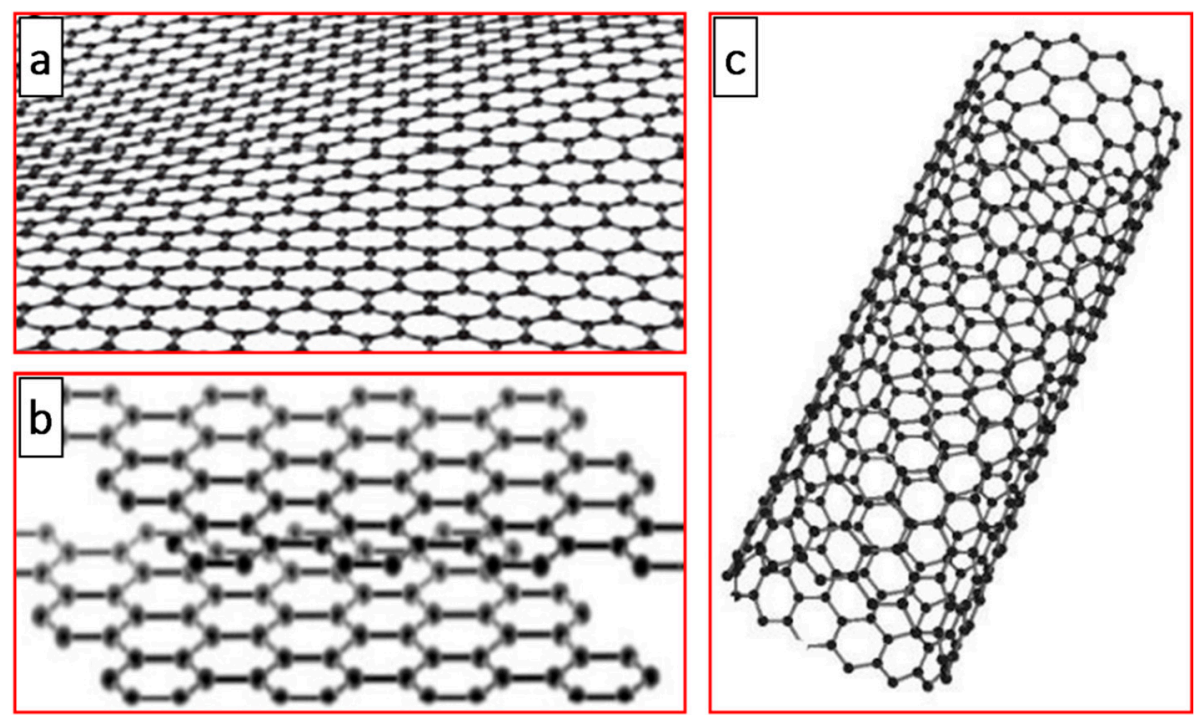

Figure 2. A conceptual model depicting the structure of graphene (a), graphite (b), and carbon nanotubes (c).

Graphene is related to graphite, and it was isolated experimentally for the first time, in 2004, from graphite [54-57]. It is a planar material with a hexagonal structure (Figure 2a), often compared to a honeycomb network, in the form of a two-dimensional (2D) crystal consisting of a simple plane of carbon atoms in $\mathrm{sp}^{2}$ hybridizations [58]. Graphene represents the basic unit of graphite, and it has a 
high optical transparency and remarkable rigidity with a tensile strength of more than 130 GPa [59]. It can be wrapped to form many other forms of carbon such as fullerenes, nanotubes, or stacked in graphite. It is important to note that the honeycomb structure of graphene gives it excellent stability with a high mobility of the electrons on its surface [59-61] and can be used in multiple applications in the fields of electronics (flexible screens, high frequency electronics, nanoelectronics, batteries, chargers), the environment (atmospheric pollution sensors, desalination of seawater), and health (targeted therapy). Generally, there are three main techniques for making graphene. The first allows us to isolate a single plane of graphene by the exfoliation of a graphite crystal using adhesive ribbon; this operation is repeated several times so that the film is sufficiently thin before depositing it on a substrate, which more often is silica [55,56,62]. The second method consists of an annealing at high temperature of a silicon carbide substrate, which induces the sublimation of $\mathrm{Si}$, consequently producing a reorganization of the carbon atoms, leading to the formation of graphene planes on the surface [63-65]. The third method is based on chemical deposition on metals from hydrocarbons [44,62,66-69]. The first technique is widely used because it is simple and inexpensive and also because the properties of graphene are easily accessible $[55,70,71]$.

The chemical properties of graphene are similar to those of graphite, and graphene can be used in multiple applications, in particular for field effect transistors due to the high mobility of the surface electrons. Because graphene has chemical stability in contact with electrolytes, mechanical elasticity and good electrical conductivity, it is widely used as a storage medium for lithium [72-75]. It is also used in gas sensors such as $\mathrm{NO}_{2}, \mathrm{CO}, \mathrm{H}_{2} \mathrm{~S}, \mathrm{H}_{2}, \mathrm{NO}, \mathrm{NH}_{3}$, and $\mathrm{CO}_{2}$ [76-85], or even as a biosensor for the detection of dopamine [86,87], $\beta$-nicotinamide adenine dinucleotide [88], paracetamol [87,89], Kojic acid [90], uric acid [86,87], vanillin [91], and caffeic acid [92]. Graphene is also used as a drug carrier [93-97] and as a charge conductor in solar cells as well as a light collector including cells and photodetectors [53,60-62,98-100]. He et al. [101] prepared a three-dimensional graphene-CNTs@Se aerogel, usingCNTs/selenium that is sandwiched between graphene nanosheets via an environmentally friendly solvothermal method. They showed that due to the 3D sandwich-type protection of the active Se, the cathode offers an initial coulombic efficiency of $92 \%$ as well as an excellent cyclic performance. This greatly facilitates the improvement of the specific energy density of Li-Se batteries [101]. Likewise, it has been shown that a graphene-CNTs-Li 2 S hybrid aerogel could be effectively used as a high capacity cathode for Li-S batteries with a reversible discharge capacity as large as $1123.6 \mathrm{~mA} \mathrm{~h} \mathrm{~g}^{-1}$, and a decrease in capacity of less than $0.02 \%$ per cycle $[102,103]$. The efficiency of these materials is attributed to the 3D structure, which is achieved by using two-dimensional (2D) graphene nanosheets and one-dimensional (1D) carbon nanotubes (CNTs), thus forming highly efficient channels for electron transfer and ionic diffusion [102,103]. This architecture was used for the development of a $\mathrm{CoS}_{2}$-carbon nanotubes-graphene anode for high performance lithium-ion batteries that can deliver an initial discharge capacity of $993 \mathrm{~mA} \mathrm{~h} \mathrm{~g}^{-1}$ at $100 \mathrm{~mA} \mathrm{~g}^{-1}$ [104].

\section{Carbon Nanotubes}

Carbon nanotubes are an allotropic form of carbon (Figure 2c), observed for the first time in 1991 by Japanese researcher Sumio Iijima during the synthesis of fullerenes by an electric arc [105]. They have been characterized as graphene sheets that are wound on themselves in the form of microtubes and will later be renamed multiwall carbon nanotubes (MWCNTs). In 1993, the synthesis of single-walled carbon nanotubes (SWCNTs) was reported by optimizing the method of synthesis by an electric arc $[106,107]$. The multisheet nanotubes consist of several graphene sheets wound concentrically with a spacing between two sheets of approximately $3.6 \AA$, slightly greater than the spacing between two sheets in graphite. The diameter of the nanotubes varies, depending on the number of sheets, from 1 to $50 \mathrm{~nm}$, and their lengths can reach $1 \mu \mathrm{m}$ [43,105,108-110]. SWCNTs consist of a graphene sheet wound on itself, and they form a tube whose diameter is between 0.4 and $3 \mathrm{~nm}$. Carbon nanotubes are therefore composed of one or more sheets of carbon atoms, as in graphite, wound on themselves forming a tube. Carbon nanotubes have good mechanical properties, good adhesion, and excellent electrical 
conductivity $[43,111-113]$. Some of these nanotubes have metallic properties $[75,114,115]$, while others are semiconductors [116-121]. SWCNTs and MWCNTs are manufactured by almost the same method; the only distinction can appear on the use of the metal catalyst, generally nickel, iron, or cobalt, which is essential for the synthesis of fullerenes. They can be prepared mainly by arc discharge, laser ablation, and chemical vapor deposition [109,120,122-126], involving one of the following as carbon precursors: xylene, acetylene, toluene, methane, benzene, etc. [43]. On the industrial level, carbon nanotubes, with the particularity that they possess, residing in a small amount of impurities, are used in many forms such as the reinforcing elements of polymers and composites, nanoporous materials for energy storage, passive (nanometric conductors) or active components (diodes and transistors), and systems allowing for the vectorization of drug molecules for the treatment of certain diseases [120-122,127-132].

\section{Activated Carbon}

Activated carbon is porous, amorphous organic material with a complex structure, characterized by high carbon content [133]. These materials include a wide range of carbonaceous substances with different properties and characteristics (porosity, specific surface, chemical nature of the surface, density, etc.). Activated carbon can be produced from any substance with a high carbon content, whether of a vegetable, fossil, or material of a synthetic nature; examples include date stones [134,135], coffee grounds [133,136,137], almond shell [138], coconut shell [139], corncob wastes [140], Acacia glauca sawdust [141], waste potato residue [142,143], rice husk [144], sunflower piths [145], tomato stem [146], banana peel [147], etc.

\subsection{Preparation of Activated Carbon}

Generally, activated carbon is prepared in two stages. The first step is the carbonization of raw material to produce a carbon surface. This step is followed by the activation by chemical oxidation or heat treatment in order to further boost the surface of the material obtained (Figure 3).

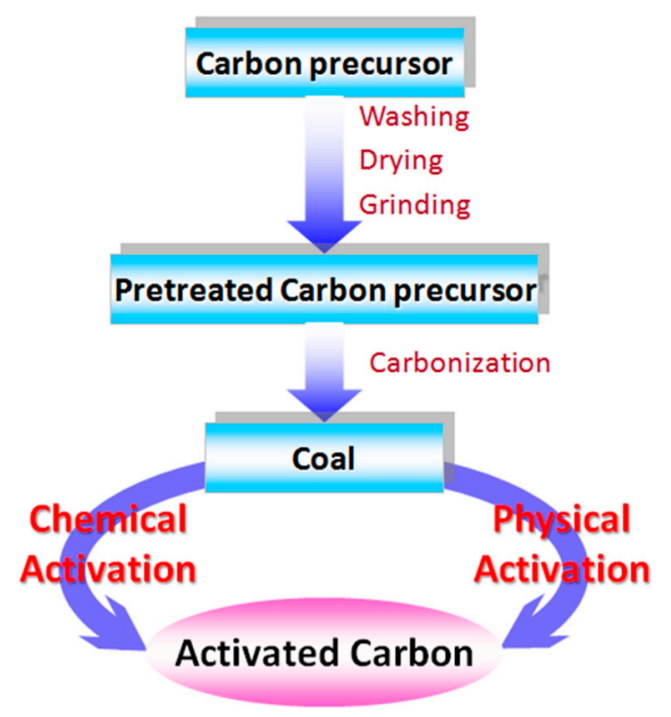

Figure 3. Steps for preparation of activated carbons.

\subsubsection{Pyrolysis}

Pyrolysis (carbonization) is the thermal decomposition of carbonaceous materials at high temperature between 400 and $800^{\circ} \mathrm{C}$, under an inert atmosphere. The resulting material is an amorphous solid rich in carbon. The remaining carbon atoms are grouped in sheets that arrange themselves irregularly, leaving gaps between them. These interstices give rise to a primary porosity of the charred product. It should be noted that the heat treatment strongly influences the texture of the materials [148]. Indeed, a gentle 
heating rate slightly modifies the carbon structure compared to the original structure of the material used and regenerates a reduced rate of volatile compounds. In the same context, the pyrolysis temperature and the residence time determine the mass loss and the nature of the surface of the biochar prepared.

The activated carbon can be obtained by the pyrolysis of carbon gels obtained by the sol-gel process. This process involves polycondensation reactions of organic precursors. In 1989, Pekala et al. introduced for the first time the synthesis of carbon aerogel by the polycondensation of resorcinol and formaldehyde in the presence of sodium carbonate as a basic catalyst $[149,150]$. Subsequently, numerous studies have vested in the preparation of polymers and activated carbon precursors by the condensation of hydroxylated benzene (phenol, catechol, 4-methylcatechol, m-cresol, hydroquinone, phloroglucinol, resorcinol, etc.) with aldehyde (formaldehyde, furfural, etc.) [151-158].

\subsubsection{Activation}

To improve the textural characteristics of the carbonate, it is necessary to subject it to an activation step. This involves opening the initial porosity and increasing accessibility to the internal structure, as well as creating new pores, eliminating the tar that clogs the pores and creating surface functions. This operation, therefore, leads to a loss of mass, and the estimation of the degree of activation can be determined by the "burn-off":

$$
\text { Burn } \left.- \text { off }(\%)=\left(\left(\mathrm{m}_{\mathrm{i}}-\mathrm{m}_{\mathrm{f}}\right) 100\right) / \mathrm{m}_{\mathrm{i}}\right)
$$

where $m_{\mathrm{i}}$ is initial mass and $m_{\mathrm{f}}$ is final mass. The burn-off provides information on the rate of activation progress and the nature of porosity. Activation can be carried out by a physical or chemical process.

\section{Physical Activation}

Physical activation consists of oxidation of biochar at high temperature $\left(>70{ }^{\circ} \mathrm{C}\right)$ under a stream of an oxidizing gas such as water vapor, oxygen, and carbon dioxide. The reactions are shown in Equations (1)-(4).

$$
\begin{gathered}
\mathrm{C}+\mathrm{H}_{2} \mathrm{O} \rightarrow \mathrm{CO}+\mathrm{H}_{2} \mathrm{C}+\mathrm{H} \\
\mathrm{C}+\mathrm{O}_{2} \rightarrow \mathrm{CO}_{2} \mathrm{C}+\mathrm{O} \\
\mathrm{C}+1 / 2 \mathrm{O}_{2} \rightarrow \mathrm{COC}+\mathrm{O} \\
\mathrm{C}+\mathrm{CO}_{2} \rightarrow 2 \mathrm{COC}+\mathrm{CO}
\end{gathered}
$$

During physical activation, the development of the porosity of the biochar depends on the nature of the oxidizing gas used, and the pores changes from micro- to mesopores and macropores $[27,31,159,160]$. The porosity is more developed in the presence of water vapor than in the presence of carbon dioxide. Indeed, the size of the water molecules is smaller than that of carbon dioxide, which gives it a greater diffusion in the pores of carbon, thus causing the enlargement of the micropores in mesopores and macropores. Generally, carbon dioxide promotes the formation of a microporous network, while water vapor generates a mesoporous or macroporous network [29,31,159-161]. Furthermore, the use of oxygen is accompanied by a significant loss in mass [27,159]. It should be noted here that physical activation is preferred because of the low cost of preparation and its simple operation, making it possible to optimize the pyrolysis step and therefore producing better control of the porosity of the activated carbon. In fact, optimization of the yield and surface properties of activated carbon lies in optimizing the temperature, the activation time, and the flow rate of the gas $\left(\mathrm{CO}_{2}\right.$, vapor, $\left.\mathrm{N}_{2}\right)[27,160]$.

\section{Chemical Activation}

The chemical activation is carried out with chemical reagents that promote dehydration and a structural reorganization of the pores of the carbonaceous material. The procedure consists of impregnating the carbonaceous material with the chemical agent then undergoing a heat treatment at a 
temperature between 400 and $900{ }^{\circ} \mathrm{C}$. Various chemical agents can be used, namely salts of alkali metals $\left(\mathrm{AlCl}_{3}, \mathrm{ZnCl}_{2}\right)$, acids $\left(\mathrm{H}_{3} \mathrm{PO}_{4}, \mathrm{H}_{2} \mathrm{SO}_{4}\right)$, or bases $\left(\mathrm{KOH}, \mathrm{NaOH}, \mathrm{K}_{2} \mathrm{CO}_{3}, \mathrm{Na}_{2} \mathrm{CO}_{3}\right)[134,140,162,163]$. Zinc chloride was the main chemical activator used on a large scale. It is now abandoned due to environmental problems linked to the toxicity of residual zinc-derived compounds. Alkali metal hydroxides $(\mathrm{KOH}, \mathrm{NaOH})$ are also used successfully to obtain a high surface area reaching $3000 \mathrm{~m}^{2} / \mathrm{g}$ [164]. Alkali metal hydroxides are often used to enlarge microporosity, while zinc chloride is used to develop strong microporosity alongside small mesoporosity. It turns out that $\mathrm{KOH}$ is the most effective activator (effective in terms of large specific surface area and high microporosity) for preparing activated carbon from biomass [31,165-167]. Furthermore, orthophosphoric acid $\left(\mathrm{H}_{3} \mathrm{PO}_{4}\right)$ is widely used in industry to impregnate lignocellulosic precursors and synthetic materials to provide active carbon with smaller pores than those activated with $\mathrm{ZnCl}_{2}$ [168]. Indeed, phosphoric acid plays both the role of an acid catalyst to favor the reactions of the depolymerization of the macromolecules that constitute biomass (cellulose, hemicellulose, and lignin) while promoting the formation of crosslinking through reactions of dehydration, cyclization, and condensation, and a combining agent that subsequently reacts with organic matter to form phosphate esters and polyphosphates, both of which serve as bridges between the fragments of biopolymers that constitute the lignocellulosic biomass $[31,169,170]$. Furthermore, the addition (or insertion) of phosphate groups leads to a process of expansion of the carbonaceous material which, after the elimination of the acid, leaves the matrix in a developed state with an accessible porous structure [31].

\subsection{Different Forms of Activated Carbon}

Activated carbon is widely used in the industry for a variety of applications [171]. Each application requires a type and form of activated carbon. The most available forms in the markets are seen in Figure 4 and also presented in the following subsections.
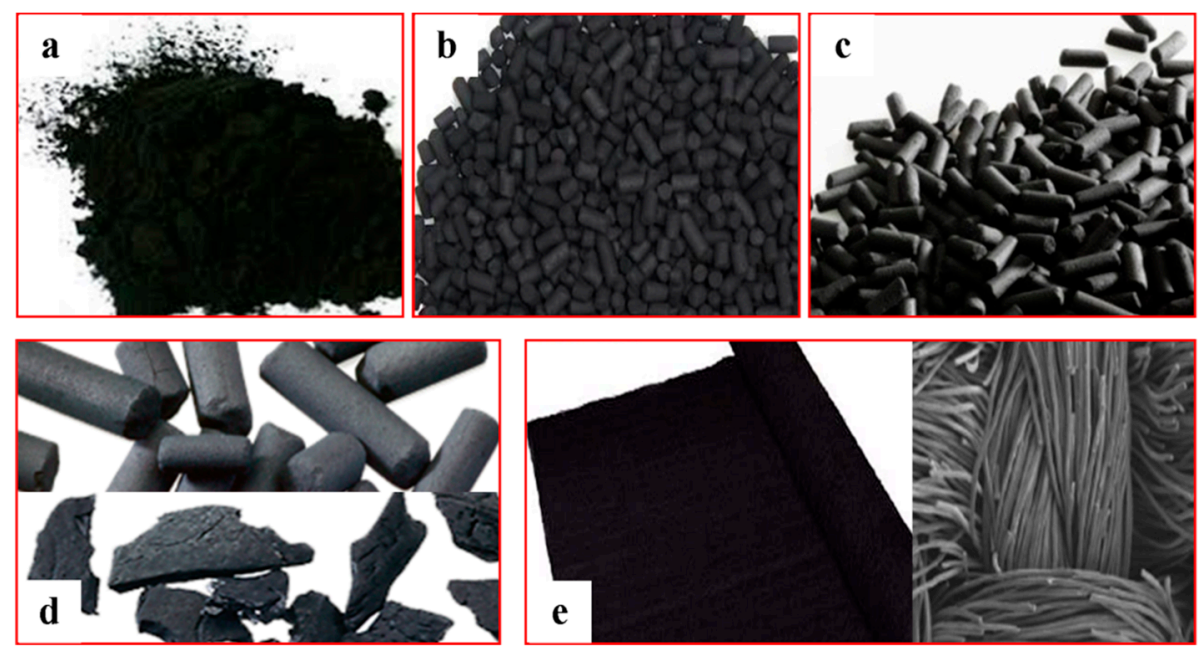

Figure 4. Forms of activated carbon: (a) activated carbon powder, (b) granular activated carbon, (c) extruded activated carbon, (d) impregnated activated carbon, (e) activated carbon fabric.

\subsubsection{Activated Carbon Powder (CAP)}

It occurs in the form of fine particles of size less than $100 \mu \mathrm{m}$ with average pore diameters of between 0.15 and $0.25 \mu \mathrm{m}$ (Figure $4 \mathrm{a}$ ). They are characterized by a large external surface and pores of reduced depths. This type of carbon is frequently used for the treatment of liquid effluents in batch mode. 


\subsubsection{Granular Activated Carbon (CAG)}

The granular or granular activated carbon is formed by grains with a size between 0.5 and $10 \mathrm{~mm}$ (Figure $4 \mathrm{~b}$ ). These grains are known by a small pore diameter, a large internal surface, and a relatively small external surface. This type of carbon is intended for the treatment of gases and liquid effluents.

\subsubsection{Extruded Activated Carbon}

To make extruded activated carbon, the carbonaceous raw material is finely ground; a binder is added to this powder in order to obtain a paste which, by an extrusion device, produces extrudates of a determined length and width (Figure 4c). These extrudates are then charred and activated under a gas flow. These materials are used for gas phase applications.

\subsubsection{Impregnated Activated Carbon}

Activated carbon can also be impregnated with a reagent to optimize its adsorption capacities for certain molecules (Figure 4d). Thus, the removal of certain impurities from gas is facilitated by this type of material, whereas it would have been impossible on a conventional carbon adsorbent.

\subsubsection{Activated Carbon Fabric (CAT)}

The active carbon tissues are obtained by the carbonization of a synthetic precursor tissue of viscose or polyacrylonitrile type followed by activation Figure 4e). These fabrics are extremely porous materials with a very high specific surface of up to $2000 \mathrm{~m}^{2} / \mathrm{g}$ and a distribution of micropores of $90 \%$ of the total pore volume. These materials are known for their medicinal applications and in catalysis.

\subsection{Application of Activated Carbon for Wastewater Treatment}

Activated carbon has long been one of the most widely used materials in the industrial sector due to its essential role in the purification of liquids and the filtration of gases. However, innovations in the manufacture and use of activated carbon are driven by the need to reuse natural resources and prevent environmental pollution. Thus, a lot of progress has been made on the optimization of these carbonaceous materials, both in terms of their manufacture and their regeneration. This has prompted several industries-for example, the metallurgical, petroleum, food, pharmaceutical, chemical, and automotive industries- to use activated carbon for various uses. Currently, the main application of activated carbon lies in the adsorption processes of pollutants in the gas or liquid phases. In fact, activated carbon is in the category of amorphous carbon, which has been chemically improved into a porous structure, mostly microporous with a surface functionality that offers active carbon the properties of excellent materials for the adsorption of various chemical species [43,172-174]. This adsorption phenomenon is due to the existence of van der Waals type physical forces, inducing a higher concentration of the adsorbate at the interface than in the fluid. Adsorption can also be caused by the formation of covalent bonds between the active sites present on the surface of the carbon and the adsorbate, which is similar to a phenomenon of chemisorption. In this regard, a limited overview is presented on the use of active carbon for the adsorption of pollutants in the liquid phase, and in particular, the field of wastewater treatment. The physicochemical surface properties of activated carbon are generally governed by the nature of the starting materials, the activation methods, and the preparation conditions (Table 1). 
Table 1. The experimental conditions for activated carbons production, specific surface area $\left(\mathrm{S}_{\mathrm{BET}}\right)$, and adsorption capacities against various toxicants.

\begin{tabular}{|c|c|c|c|c|c|c|c|c|}
\hline Carbon Precursor & $\begin{array}{l}\text { Activ. Temp./Activ. } \\
\text { Time }\end{array}$ & Activation & Reagent & $S_{\mathrm{BET}}\left(\mathrm{m}^{2} / \mathrm{g}\right)$ & Dose $(g / L)$ & Pollutant & $\begin{array}{c}Q_{\max }(\mathrm{mg} / \mathrm{g}) / C_{0} \\
(\mathrm{mg} / \mathrm{L})\end{array}$ & Ref. \\
\hline Acacia erioloba & $600{ }^{\circ} \mathrm{C} / 1 \mathrm{~h}$ & Chemical & $\mathrm{H}_{2} \mathrm{SO}_{4}$ & 10.4 & 1 & $\mathrm{MB}$ & $1.5 / 25-500$ & [175] \\
\hline Acacia glauca & $450^{\circ} \mathrm{C} / 1 \mathrm{~h}$ & Chemical & $\mathrm{H}_{3} \mathrm{PO}_{4}$ & 311.2 & 2 & 4-NP & $204.7 / 50-500$ & [141] \\
\hline Acrylic fibrous waste & $1200{ }^{\circ} \mathrm{C}$ & Physical & $\mathrm{O}_{2}$ & 280 & 2 & $\mathrm{MB}$ & $8.7 / 2-10$ & [176] \\
\hline Apricot shell & $400{ }^{\circ} \mathrm{C} / 1.5 \mathrm{~h}$ & Chemical & $\mathrm{H}_{3} \mathrm{PO}_{4}$ & 307 & - & Tetracycline & 76.9 (FBA) & [177] \\
\hline \multirow{2}{*}{ Ashitaba waste } & \multirow{2}{*}{$800^{\circ} \mathrm{C} / 1.5 \mathrm{~h}$} & \multirow{2}{*}{ Chemical } & \multirow{2}{*}{$\mathrm{ZnCl}_{2}$} & \multirow{2}{*}{1228.5} & 0.01 & Congo Red & $345.1 / 0-100$ & \multirow{2}{*}{178} \\
\hline & & & & & 0.01 & $\mathrm{MB}$ & $289.2 / 0-50$ & \\
\hline Bamboo & $600^{\circ} \mathrm{C} / 4 \mathrm{~h}$ & Chemical & $\mathrm{H}_{3} \mathrm{PO}_{4}$ & 1400 & 1 & Reactive Black 5 & 489.9/10-1000 & [179] \\
\hline Bamboo waste + Ms & $550{ }^{\circ} \mathrm{C} / 0.5 \mathrm{~h}$ & Chemical & $\mathrm{ZnCl}_{2}$ & 289.5 & 8 & Phenol & $25.3 / 227.5$ & [180] \\
\hline \multirow{3}{*}{ Banana peels } & MW 700W/10 min & Chemical & $\mathrm{KOH} / \mathrm{NaOH}$ & 1038 & 2.5 & MG & $22.5 / 10$ & [147] \\
\hline & \multirow{2}{*}{$800^{\circ} \mathrm{C} / 0.5 \mathrm{~h}$} & \multirow{2}{*}{ Chemical } & \multirow{2}{*}{$\mathrm{K}_{2} \mathrm{CO}_{3}$} & \multirow{2}{*}{1188} & \multirow{2}{*}{0.4} & Me-Red & $400 / 40-220$ & \multirow{2}{*}{181} \\
\hline & & & & & & $\mathrm{MB}$ & $454.5 / 40-220$ & \\
\hline Banyan tree & $500^{\circ} \mathrm{C} / 1 \mathrm{~h}$ & Chemical & $\mathrm{KOH}$ & 988 & 2.5 & Phenol & 26.9/20-100 & [182] \\
\hline Bermuda grass & $450^{\circ} \mathrm{C} / 2 \mathrm{~h}$ & Chemical & $\mathrm{KOH}$ & 1833.6 & 0.5 & $\mathrm{Cr}(\mathrm{VI})$ & $403.2 / 0-200$ & [183] \\
\hline \multirow{2}{*}{ Black cumin seeds } & \multirow{2}{*}{$40^{\circ} \mathrm{C} / 48 \mathrm{~h}$} & \multirow{2}{*}{ Chemical } & \multirow{2}{*}{$\mathrm{H}_{2} \mathrm{SO}_{4}$} & \multirow{2}{*}{-} & 2 & $\mathrm{MB}$ & $16.8 / 100$ & \multirow{2}{*}{184} \\
\hline & & & & & 2 & $\mathrm{~Pb}^{2+}$ & $17.9 / 100$ & \\
\hline Black wattle bark waste & $700{ }^{\circ} \mathrm{C} / 2 \mathrm{~h}$ & Chemical & $\mathrm{ZnCl}_{2}$ & 414 & 1 & Phenol & $85.7 / 50-500$ & [185] \\
\hline Caesalpinia ferrea & $600^{\circ} \mathrm{C}$ & Chemical & $\mathrm{ZnCl}_{2}$ & 1480 & 1.5 & Captopril & $535 / 120-2500$ & [186] \\
\hline Carya illinoinensis & $700{ }^{\circ} \mathrm{C} / 2 \mathrm{~h}$ & $\begin{array}{c}\text { Chemical + } \\
\text { Physical }\end{array}$ & $\begin{array}{c}\mathrm{NaCl}, \mathrm{CaCl}_{2} \\
\mathrm{KCl} / \mathrm{CO}_{2}\end{array}$ & $490-808$ & 2 & $\begin{array}{l}\mathrm{Cu}^{2+}, \mathrm{Cd}^{2+} \\
\mathrm{Ni}^{2+}, \mathrm{Zn}^{2+}\end{array}$ & $\begin{array}{c}0.011-0.368 \\
\mathrm{mmol} / \mathrm{g}\end{array}$ & [187] \\
\hline \multirow{2}{*}{ Chamerion angustifolium } & \multirow{2}{*}{$450{ }^{\circ} \mathrm{C} / 0.5 \mathrm{~h}$} & \multirow{2}{*}{ Physical } & \multirow{2}{*}{-} & \multirow{2}{*}{117.7} & \multirow{2}{*}{0.8} & $\mathrm{Co}^{2+}$ & $9.8 / 1-40$ & \multirow{2}{*}{ [188] } \\
\hline & & & & & & $\mathrm{Ni}^{2+}$ & $10.1 / 1-40$ & \\
\hline \multirow{2}{*}{ Citrus limetta peels } & $250{ }^{\circ} \mathrm{C} / 2 \mathrm{~h}$ & Chemical & $\mathrm{FeCl}_{3}$ & - & 1 & $\mathrm{~F}^{-}$ & $4.9 / 5-30$ & \multirow{2}{*}{ [28] } \\
\hline & $500{ }^{\circ} \mathrm{C} / 2 \mathrm{~h}$ & Chemical & $\mathrm{FeCl}_{3}$ & - & 1 & $\mathrm{~F}^{-}$ & $9.7 / 5-30$ & \\
\hline
\end{tabular}


Table 1. Cont.

\begin{tabular}{|c|c|c|c|c|c|c|c|c|}
\hline Carbon Precursor & $\begin{array}{l}\text { Activ. Temp./Activ. } \\
\text { Time }\end{array}$ & Activation & Reagent & $S_{\text {BET }}\left(\mathrm{m}^{2} / \mathrm{g}\right)$ & Dose $(g / L)$ & Pollutant & 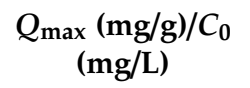 & Ref. \\
\hline Coconut husk & $350^{\circ} \mathrm{C} / 3 \mathrm{~h}$ & Chemical & $\mathrm{KOH}$ & 1448 & - & $\mathrm{F}^{-}$ & 6.7 (FBA) & [139] \\
\hline Coconut shell & $600^{\circ} \mathrm{C} / 1 \mathrm{~h}$ & Chemical & $\mathrm{NaOH}$ & 876 & 1 & $\mathrm{MB}$ & $200 / 25-250$ & [189] \\
\hline \multirow{2}{*}{ Cocos nucifera shell } & \multirow{2}{*}{$275^{\circ} \mathrm{C} / 2 \mathrm{~h}$} & \multirow{2}{*}{$\begin{array}{l}\text { Physico } \\
\text { chemical }\end{array}$} & \multirow{2}{*}{$\mathrm{ZnCl}_{2} / \mathrm{H}_{2} \mathrm{O}$} & \multirow{2}{*}{1652} & \multirow{2}{*}{1} & Phenol Red & $351 / 500$ & \multirow{2}{*}{ [190] } \\
\hline & & & & & & Phenol & $264 / 500$ & \\
\hline \multirow{4}{*}{ Coffee grounds } & \multirow{2}{*}{$800^{\circ} \mathrm{C} / 1 \mathrm{~h}$} & \multirow{2}{*}{$\begin{array}{l}\text { Physico } \\
\text { chemical }\end{array}$} & \multirow{2}{*}{$\mathrm{KOH} / \mathrm{CO}_{2}$} & \multirow{2}{*}{1865} & 1 & Phenol & $211 / 10-200$ & \multirow{2}{*}{ [136] } \\
\hline & & & & & 1 & MB & $390 / 50-500$ & \\
\hline & \multirow{2}{*}{$950^{\circ} \mathrm{C} / 3 \mathrm{~h}$} & \multirow{2}{*}{ Physical } & \multirow{2}{*}{$\mathrm{CO}_{2}$} & \multirow{2}{*}{2407.8} & 0.66 & MB & $680 / 20-150$ & \multirow{2}{*}{ [29] } \\
\hline & & & & & 0.66 & $\mathrm{MO}$ & $612 / 20-150$ & \\
\hline Coffee husk & \multirow{2}{*}{$800^{\circ} \mathrm{C} / 0.5 \mathrm{~h}$} & \multirow{2}{*}{ Physical } & \multirow{2}{*}{$\mathrm{H}_{2} \mathrm{O}$} & 383 & 1 & \multirow{2}{*}{$\mathrm{Ni}^{2+}$} & $57.1 / 30-120$ & \multirow{2}{*}{ [191] } \\
\hline Coffee spent & & & & 464 & 0.8 & & $51.9 / 30-120$ & \\
\hline Corncob & $400{ }^{\circ} \mathrm{C} / 1.5 \mathrm{~h}$ & Chemical & $\mathrm{H}_{3} \mathrm{PO}_{4}$ & 1097 & 2 & $\mathrm{NH}^{4+}$ & $17 / 10-40$ & [140] \\
\hline Corncob & $800^{\circ} \mathrm{C} / 1 \mathrm{~h}$ & Chemical & $\mathrm{KOH}$ & 1054.2 & 0.02 & $\mathrm{Hg}(\mathrm{II})$ & $2.3 / 0.02-0.1$ & [192] \\
\hline Corncob & $800{ }^{\circ} \mathrm{C} / 1.5 \mathrm{~h}$ & Chemical & $\mathrm{NaOH}$ & 2381 & 0.2 & $\mathrm{~Pb}^{2+}$ & $381 / 10-500$ & [193] \\
\hline Corncob & $892^{\circ} \mathrm{C} / 40 \mathrm{~min}$ & Physical & $\mathrm{H}_{2} \mathrm{O}$ & - & 0.4 & Roxarsone & $309.6 / 40-350$ & [194] \\
\hline Date palm petiole & $600^{\circ} \mathrm{C} / 2 \mathrm{~h}$ & Chemical & $\mathrm{NaOH}$ & 655 & 4 & Indigo carmine & $53.7 / 10-200$ & [135] \\
\hline \multirow{2}{*}{ Durian shell } & \multirow{2}{*}{$900{ }^{\circ} \mathrm{C} / 15 \mathrm{~min}$} & \multirow{2}{*}{ Physical } & \multirow{2}{*}{$\mathrm{CO}_{2}$} & \multirow{2}{*}{917} & 10 & AMX & $142.7 / 250$ & \multirow{2}{*}[195]{} \\
\hline & & & & & 10 & Tetracycline & $126 / 250$ & \\
\hline Eucalyptus residue & $400{ }^{\circ} \mathrm{C} / 3 \mathrm{~h}$ & Chemical & $\mathrm{H}_{3} \mathrm{PO}_{4}$ & 1545 & 0.2 & $\mathrm{MB}$ & $977 / 140-260$ & [196] \\
\hline Eucalyptus sawdust & $700^{\circ} \mathrm{C} / 75 \mathrm{~min}$ & Chemical & $\mathrm{FeCl}_{3}$ & 645.2 & 1 & $\mathrm{MB}$ & $162.8 / 50-500$ & [197] \\
\hline \multirow{3}{*}{ Fox nutshell } & \multirow{2}{*}{$600^{\circ} \mathrm{C} / 1 \mathrm{~h}$} & \multirow{3}{*}{ Chemical } & \multirow{2}{*}{$\mathrm{ZnCl}_{2}$} & 2869 & 5 & Phenol & 75.3 & [198] \\
\hline & & & & & 0.4 & $\mathrm{MB}$ & 968.7 & \\
\hline & $700{ }^{\circ} \mathrm{C} / 1 \mathrm{~h}$ & & $\mathrm{H}_{3} \mathrm{PO}_{4}$ & 2636 & 0.4 & $\mathrm{Cr}(\mathrm{VI})$ & $74.9 / 35$ & [199] \\
\hline
\end{tabular}


Table 1. Cont.

\begin{tabular}{|c|c|c|c|c|c|c|c|c|}
\hline Carbon Precursor & $\begin{array}{l}\text { Activ. Temp./Activ. } \\
\text { Time }\end{array}$ & Activation & Reagent & $S_{\text {BET }}\left(\mathrm{m}^{2} / \mathrm{g}\right)$ & Dose $(g / L)$ & Pollutant & $\begin{array}{c}Q_{\max }(\mathrm{mg} / \mathrm{g}) / C_{0} \\
(\mathrm{mg} / \mathrm{L})\end{array}$ & Ref. \\
\hline \multirow{2}{*}{ Grape waste } & \multirow{2}{*}{$600^{\circ} \mathrm{C} / 1 \mathrm{~h}$} & \multirow{2}{*}{ Chemical } & \multirow{2}{*}{$\mathrm{ZnCl}_{2.5}$} & \multirow{2}{*}{1455} & \multirow{2}{*}{0.6} & MY & $386 / 200-900$ & \multirow{2}{*}{ [200] } \\
\hline & & & & & & $\mathrm{MB}$ & $417 / 200-900$ & \\
\hline \multirow{2}{*}{ Grapefruit peels } & \multirow{2}{*}{$800^{\circ} \mathrm{C} / 0.5 \mathrm{~h}$} & \multirow{2}{*}{ Chemical } & \multirow{2}{*}{$\mathrm{K}_{2} \mathrm{CO}_{3}$} & \multirow{2}{*}{1198} & \multirow{2}{*}{0.4} & Me-Red & $454.5 / 40-220$ & \multirow{2}{*}{ [181] } \\
\hline & & & & & & MB & $456.2 / 40-220$ & \\
\hline Guava seeds & $750{ }^{\circ} \mathrm{C} / 1.5 \mathrm{~h}$ & Chemical & $\mathrm{NaOH}$ & 2573.6 & 1 & AMX & $570.4 / 400-800$ & [162] \\
\hline Jackfruit-PPI-1 & $600^{\circ} \mathrm{C} / 1 \mathrm{~h}$ & Chemical & $\mathrm{K}_{2} \mathrm{CO}_{3}$ & 987 & 1 & Disperse Blue 14 & $178.5 / 10-120$ & [201] \\
\hline Maghara coal & $550{ }^{\circ} \mathrm{C} / 1.5 \mathrm{~h}$ & Chemical & $\mathrm{NaOH}$ & 49 & 0.5 & $\mathrm{MB}$ & $28 / 10-50$ & [202] \\
\hline \multirow{2}{*}{ Mandarin peels } & \multirow{2}{*}{$800^{\circ} \mathrm{C} / 0.5 \mathrm{~h}$} & \multirow{2}{*}{ Chemical } & \multirow{2}{*}{$\mathrm{K}_{2} \mathrm{CO}_{3}$} & \multirow{2}{*}{1077} & \multirow{2}{*}{0.4} & Me-Red & $357.1 / 40-220$ & \multirow{2}{*}{181} \\
\hline & & & & & & $\mathrm{MB}$ & $400 / 40-220$ & \\
\hline Olive stones & $500^{\circ} \mathrm{C} / 3 \mathrm{~h}$ & Physical & $\mathrm{N}_{2}$ & 9.1 & 1 & MB & 769/50-1000 & [203] \\
\hline Opuntia ficus-indica & $600^{\circ} \mathrm{C} / 2 \mathrm{~h}$ & Chemical & $\mathrm{NaOH}$ & 331.5 & 6 & 4-NP & $16.1 / 10-150$ & [204] \\
\hline \multirow{5}{*}{ Palm shell } & \multirow{5}{*}{$900{ }^{\circ} \mathrm{C} / 1 \mathrm{~h}$} & \multirow{5}{*}{ Chemical } & \multirow{5}{*}{$\begin{array}{c}\mathrm{H}_{2} \mathrm{SO}_{4}+ \\
\mathrm{K}_{2} \mathrm{~S}_{2} \mathrm{O}_{8}\end{array}$} & \multirow{5}{*}{770} & \multirow{5}{*}{-} & Bis-A & 45.45 & \multirow{5}{*}[205]{} \\
\hline & & & & & & 4-ClP & 163.9 & \\
\hline & & & & & & 4-NP & 166.7 & \\
\hline & & & & & & 2,4-DNP & 250.0 & \\
\hline & & & & & & $2,4,6-\mathrm{TNP}$ & 142.0 & \\
\hline \multirow{4}{*}{ PAN } & \multirow{4}{*}{$800{ }^{\circ} \mathrm{C} / 0.5 \mathrm{~h}$} & \multirow{4}{*}{ Chemical } & \multirow{2}{*}{$\mathrm{K}_{2} \mathrm{CO}_{3}$} & \multirow{2}{*}{2217} & \multirow{2}{*}{-} & MCPA & $4.2 \mathrm{mmol} / \mathrm{g}$ & \\
\hline & & & & & & $2,4-\mathrm{D}$ & $3.4 \mathrm{mmol} / \mathrm{g}$ & [206] \\
\hline & & & $\mathrm{KOH}$ & 2828 & - & MCPA & $5.1 \mathrm{mmol} / \mathrm{g}$ & \\
\hline & & & & & & $2,4-\mathrm{D}$ & $4.4 \mathrm{mmol} / \mathrm{g}$ & \\
\hline $\begin{array}{c}\text { Parthenium } \\
\text { hysterophorus }\end{array}$ & $500^{\circ} \mathrm{C} / 2 \mathrm{~h}$ & Chemical & $\mathrm{H}_{3} \mathrm{PO}_{4}$ & 256.5 & 90 & $\mathrm{Cr}$ & $0.7 / 40-100$ & [207] \\
\hline
\end{tabular}


Table 1. Cont.

\begin{tabular}{|c|c|c|c|c|c|c|c|c|}
\hline Carbon Precursor & $\begin{array}{l}\text { Activ. Temp./Activ. } \\
\text { Time }\end{array}$ & Activation & Reagent & $S_{\text {BET }}\left(\mathrm{m}^{2} / \mathrm{g}\right)$ & Dose $(g / L)$ & Pollutant & 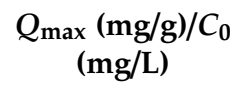 & Ref. \\
\hline Peanut shells & $700^{\circ} \mathrm{C} / 3 \mathrm{~h}$ & Chemical & $\mathrm{H}_{2} \mathrm{SO}_{4}$ & 570 & 20 & $\begin{array}{c}2,4-\mathrm{DNT}-3-\mathrm{SO} 3 \\
+ \\
2,4-\mathrm{DNT}-5-\mathrm{SO} 3\end{array}$ & 32 & [208] \\
\hline \multirow{4}{*}{ PET } & \multirow{4}{*}{$800{ }^{\circ} \mathrm{C} / 0.5 \mathrm{~h}$} & \multirow{4}{*}{ Chemical } & \multirow{2}{*}{$\mathrm{K}_{2} \mathrm{CO}_{3}$} & \multirow{2}{*}{1206} & - & MCPA & $2.4 \mathrm{mmol} / \mathrm{g}$ & \multirow{4}{*}[206]{} \\
\hline & & & & & - & $2,4-\mathrm{D}$ & $1.9 \mathrm{mmol} / \mathrm{g}$ & \\
\hline & & & \multirow{2}{*}{$\mathrm{KOH}$} & \multirow{2}{*}{1439} & - & MCPA & $2.6 \mathrm{mmol} / \mathrm{g}$ & \\
\hline & & & & & - & $2,4-\mathrm{D}$ & $2.5 \mathrm{mmol} / \mathrm{g}$ & \\
\hline \multirow{4}{*}{ Phoenix dactylifera $\mathrm{L}$. } & $500{ }^{\circ} \mathrm{C} / 1 \mathrm{~h}$ & Chemical & $\mathrm{KOH}$ & 817 & 0.15 & Levofloxacin & $101.3 / 50-250$ & {$[134]$} \\
\hline & $300^{\circ} \mathrm{C} / 0.5 \mathrm{~h}$ & Chemical & $\mathrm{H}_{3} \mathrm{PO}_{4}$ & 1225.5 & 5.25 & Rh-B & 196 & [209] \\
\hline & \multirow{2}{*}{$500^{\circ} \mathrm{C} / 1 \mathrm{~h}$} & \multirow{2}{*}{ Chemical } & \multirow{2}{*}{$\mathrm{K}_{2} \mathrm{CO}_{3}$} & \multirow{2}{*}{852} & \multirow{2}{*}{-} & Ciprofloxacin & 2 (FBA) & \multirow{2}{*}{ [210] } \\
\hline & & & & & & Norfloxacin & 1.9 (FBA) & \\
\hline Pinus eldarica & $750{ }^{\circ} \mathrm{C} / 3 \mathrm{~h}$ & Chemical & $\mathrm{K}_{2} \mathrm{CO}_{3}$ & 1201 & 0.1 & SDS & $347 / 10-125$ & [211] \\
\hline \multirow{2}{*}{ Pomelo peels } & \multirow{2}{*}{$800^{\circ} \mathrm{C} / 0.5 \mathrm{~h}$} & \multirow{2}{*}{ Chemical } & \multirow{2}{*}{$\mathrm{K}_{2} \mathrm{CO}_{3}$} & \multirow{2}{*}{836} & \multirow{2}{*}{0.4} & Me-Red & $208.3 / 40-220$ & \multirow{2}{*}{ [181] } \\
\hline & & & & & & MB & $222.2 / 40-220$ & \\
\hline Potato peels & $400^{\circ} \mathrm{C} / 1 \mathrm{~h}$ & Chemical & $\mathrm{H}_{3} \mathrm{PO}_{4}$ & 904.5 & 0.5 & Bis-A & $445.9 / 0-300$ & [212] \\
\hline Potato peels & $500^{\circ} \mathrm{C} / 0.5 \mathrm{~h}$ & Chemical & $\mathrm{H}_{3} \mathrm{PO}_{4}$ & 676 & 10 & $\mathrm{~Pb}^{2+}$ & $8.9 / 100$ & [143] \\
\hline \multirow{3}{*}{$\begin{array}{l}\text { Residue from biomass } \\
\text { gasification }\end{array}$} & \multirow{3}{*}{$600{ }^{\circ} \mathrm{C} / 1 \mathrm{~h}$} & \multirow{3}{*}{ Chemical } & \multirow{3}{*}{$\mathrm{ZnCl}_{2}$} & \multirow{3}{*}{259} & \multirow{3}{*}{5} & $\mathrm{Fe}^{2+}$ & $20.5 / 25-125$ & \multirow{3}{*}[213]{} \\
\hline & & & & & & $\mathrm{Cu}^{2+}$ & $23.1 / 25-125$ & \\
\hline & & & & & & $\mathrm{Ni}^{2+}$ & $18.2 / 25-125$ & \\
\hline Rice husk & $950{ }^{\circ} \mathrm{C} / 1 \mathrm{~h}$ & Chemical & $\mathrm{K}_{2} \mathrm{CO}_{3}$ & 1330 & 1 & $\mathrm{NO}^{3-}$ & $8.1 / 15$ & [144] \\
\hline Rice husk & $800^{\circ} \mathrm{C} / 1.5 \mathrm{~h}$ & Chemical & $\mathrm{NaOH}$ & 2786 & 0.2 & $\mathrm{~Pb}^{2+}$ & $492 / 10-500$ & [193] \\
\hline Sargassum fusiforme & $900{ }^{\circ} \mathrm{C} / 0.5 \mathrm{~h}$ & Physical & $\mathrm{CO}_{2}$ & 1329 & 0.8 & Congo Red & $234 / 50-200$ & [159] \\
\hline Sewage sludge & $500{ }^{\circ} \mathrm{C} / 1 \mathrm{~h}$ & Chemical & $\mathrm{ZnCl}_{2}$ & 721 & 0.1 & $\begin{array}{c}\text { FL, ANT, PYR, } \\
\text { BeP, OP, NP, } \\
\text { DBP, DEHP }\end{array}$ & $\begin{array}{l}0.07-2.8 / \\
0.01-0.3\end{array}$ & [214] \\
\hline
\end{tabular}


Table 1. Cont.

\begin{tabular}{|c|c|c|c|c|c|c|c|c|}
\hline Carbon Precursor & $\begin{array}{l}\text { Activ. Temp./Activ. } \\
\text { Time }\end{array}$ & Activation & Reagent & $S_{\text {BET }}\left(\mathrm{m}^{2} / \mathrm{g}\right)$ & Dose $(g / L)$ & Pollutant & $\begin{array}{c}Q_{\max }(\mathrm{mg} / \mathrm{g}) / C_{0} \\
(\mathrm{mg} / \mathrm{L})\end{array}$ & Ref. \\
\hline \multirow{2}{*}{$\begin{array}{c}\text { Sugarcane } \\
\text { bagasse }\end{array}$} & $550{ }^{\circ} \mathrm{C} / 0.5 \mathrm{~h}$ & Chemical & $\mathrm{ZnCl}_{2}$ & 182.9 & 0.2 & $\mathrm{Hg}^{2+}$ & $11.5 / 1$ & \multirow{2}{*}{ [30] } \\
\hline & $300^{\circ} \mathrm{C} / 1 \mathrm{~h}$ & Chemical & $\mathrm{H}_{3} \mathrm{PO}_{4}$ & 7.4 & 0.2 & $\mathrm{Hg}^{2+}$ & $13.6 / 1$ & \\
\hline \multirow{2}{*}{ Sunflower piths } & \multirow{2}{*}{$700{ }^{\circ} \mathrm{C} / 1 \mathrm{~h}$} & \multirow{2}{*}{ Chemical } & $\mathrm{NaOH}$ & 2690 & 1 & $\mathrm{MB}$ & $965 / 50-1000$ & \multirow{2}{*}{ [145] } \\
\hline & & & $\mathrm{KOH}$ & 2090 & 1 & MB & $580 / 50-1000$ & \\
\hline Syzygium cumini seed & $900^{\circ} \mathrm{C} / 1 \mathrm{~h}$ & Chemical & $\mathrm{KOH}$ & 747 & 6 & $\mathrm{~F}^{-}$ & $1.2 / 20.04$ & [215] \\
\hline Tomato stem & $700{ }^{\circ} \mathrm{C} / 1 \mathrm{~h}$ & Chemical & $\mathrm{FeCl}_{2}$ & 971 & 1 & Congo red & $158.7 / 100-500$ & [146] \\
\hline \multirow{2}{*}{ Tomato waste } & \multirow{2}{*}{$600^{\circ} \mathrm{C} / 1 \mathrm{~h}$} & \multirow{2}{*}{ Chemical } & \multirow{2}{*}{$\mathrm{ZnCl}_{2}$} & \multirow{2}{*}{1093} & \multirow{2}{*}{0.6} & MY & $385 / 200-900$ & \multirow{2}{*}[216]{} \\
\hline & & & & & & $\mathrm{MB}$ & 400/200-900 & \\
\hline Tyre & $750^{\circ} \mathrm{C}$ & Chemical & $\mathrm{KOH}$ & 700 & 0.5 & Bis-A & $123 / 5-80$ & [217] \\
\hline \multirow{4}{*}{ Ulva lactuca } & \multirow{4}{*}{$800^{\circ} \mathrm{C} / 3 \mathrm{~h}$} & \multirow{4}{*}{ Chemical } & \multirow{4}{*}{$\mathrm{KOH}$} & \multirow{4}{*}{345.4} & \multirow{4}{*}{0.06} & $\mathrm{Cu}^{2+}$ & $84.7 / 5-80$ & \multirow{4}{*}{163} \\
\hline & & & & & & $\mathrm{Cd}^{2+}$ & $84.6 / 5-80$ & \\
\hline & & & & & & $\mathrm{Cr}^{3+}$ & $82 / 5-80$ & \\
\hline & & & & & & $\mathrm{Pb}^{2+}$ & $83.3 / 5-80$ & \\
\hline \multirow{2}{*}{ Walnut shell } & \multirow{2}{*}{$900^{\circ} \mathrm{C} / 1.5 \mathrm{~h}$} & \multirow{2}{*}{ Chemical } & \multirow{2}{*}{$\mathrm{ZnCl}_{2}$} & \multirow{2}{*}{1626.9} & 0.01 & Congo Red & $281.4 / 0-100$ & \multirow{2}{*}{ [178] } \\
\hline & & & & & 0.01 & $\mathrm{MB}$ & $314.1 / 0-50$ & \\
\hline Waste carpets & $700^{\circ} \mathrm{C} / 4 \mathrm{~h}$ & Chemical & $\mathrm{H}_{3} \mathrm{PO}_{4}$ & 953 & 8 & $\mathrm{MB}$ & $769.2 / 50-800$ & [218] \\
\hline \multirow{3}{*}{ Waste tires } & \multirow{3}{*}{$750^{\circ} \mathrm{C}$} & \multirow{3}{*}{ Chemical } & \multirow{3}{*}{$\mathrm{KOH}$} & \multirow{3}{*}{265} & 0.3 & $\mathrm{~Pb}^{2+}$ & 49.7/5-100 & \multirow{3}{*}[219]{} \\
\hline & & & & & 0.3 & $\mathrm{Cd}^{2+}$ & $10.4 / 5-100$ & \\
\hline & & & & & 0.3 & $\mathrm{Cr}^{3+}$ & $29.4 / 5-100$ & \\
\hline White sugar & $700{ }^{\circ} \mathrm{C} / 2 \mathrm{~h}$ & Chemical & $\mathrm{NaOH}$ & 1144.7 & 0.8 & Rh-B & $123.4 / 25-100$ & [220] \\
\hline
\end{tabular}


As a result, the adsorption properties of activated carbon vary from source to source. Biomass is widely used for the production of activated carbon because of its great abundance, its renewability, and its low cost. In fact, various biomass wastes have been used to produce adsorbents by chemical or physical activation. Nowicki et al. [181] studied the influence of the precursor on the structural and sorption properties of methyl red (Me-Red) and methylene blue (MB)of activated carbon obtained by chemical activation with potassium carbonate involving grapefruit, mandarin, pomelo, and banana peels as precursors. They confirmed that this waste can be successfully used as precursors for the preparation of activated carbon that show a well-developed porous structure with specific surfaces of $836,1198,1077$, and $1188 \mathrm{~m}^{2} / \mathrm{g}$, respectively, optimizing the conditions activation (temperature, time, impregnation ratio). It turns out that the efficiency of removal of Me-Red and MB from aqueous solutions depends on the type of precursor used and that the adsorption capacity increases with the increase in the specific surface area of the adsorbent. Charcoal from grapefruit peels was found to be the most effective adsorbent with a sorption capacity of $456.2 \mathrm{and} 454.5 \mathrm{mg} / \mathrm{g}$ for $\mathrm{MB}$ and Me-Red, respectively. In the same context, Baysal et al. [145] found that the adsorption capacity of MB increases from 580 to $965 \mathrm{mg} / \mathrm{g}$ when the surface area of activated carbon obtained from sunflower piths increases from 2090 to $2690 \mathrm{~m}^{2} / \mathrm{g}$. The increase in specific surface area was attributed to the nature of the activating agent where the highest value was obtained with sodium hydroxide [138]. Activation by potassium hydroxide mainly caused the development of micropores with a very narrow pore distribution. Thus, the sodium hydroxide appeared to be more reactive, which resulted in the micropores fusing to form mesopores $[135,145,193,204]$. Indeed, several studies have reported that potassium hydroxide is an activator that promotes the formation of activated carbon with a microporous structure $[166,167,183,206,221]$. However, some authors obtained a mesoporous structure by activation with potassium hydroxide [134]. Indeed, the structural properties of activated carbon also depend on the temperature and the duration of activation as well as the impregnation ratio. For example, the activated carbon obtained from a near of corn passes from a macroporous structure for a pyrolysis temperature of $800{ }^{\circ} \mathrm{C}$ and duration of $1 \mathrm{~h} \mathrm{[192]} \mathrm{to} \mathrm{a} \mathrm{microporous} \mathrm{structure} \mathrm{for} \mathrm{a}$ pyrolysis temperature of $850{ }^{\circ} \mathrm{C}$ and duration of $3 \mathrm{~h}$ [221]. Likewise, the specific surface increases from 1054 to $1618 \mathrm{~m}^{2} / \mathrm{g}$ [192,221]. In this respect, the variation in the parameters of preparation of the activated carbon offers varied in the textural and structural properties. Consequently, there is a variation of the porosity, both in shape and in dimensions, of the functional groups and of the $\mathrm{pH}_{\mathrm{ZCN}}$. These properties with the conditions of application determine the applicability of the activated carbon. Large-pore activated carbon is effectively used for the adsorption of large pollutants and vice versa $[135,179,204,206]$.

Da Paixao Cansado et al. [206] did a comparative study of the adsorption of pesticides on chemically prepared activated carbon involving $\mathrm{K}_{2} \mathrm{CO}_{3}$ and $\mathrm{KOH}$. The aim was to obtain activated carbon from polymer waste (Polyacrylonitrile (PAN) and Polyethyleneterephthalate (PET)) and to determine their main characteristics, namely the characterization of their porosity, their functional groups on the surface, and their adsorption capacities for 4-chloro-2-methylphenoxyacetic acid (MCPA) and 2,4-dichlorophenoxyacetic acid (2,4-D). The results showed that these pollutants were better adsorbed on the activated carbon obtained with $\mathrm{KOH}$, which has a microporosity and a specific surface area greater than that obtained with $\mathrm{K}_{2} \mathrm{CO}_{3}$ [206]. In the same context, Laksaci et al. [136] studied the influence of the combination of $\mathrm{CO}_{2}$ with $\mathrm{KOH}$ on the morphological properties and the sorption capacities of adsorbents from coffee grounds for phenol and $\mathrm{MB}$. Activation by $\mathrm{KOH}$ under $\mathrm{CO}_{2}$ flow considerably improves the porous structure of the adsorbents. Indeed, the use of $\mathrm{CO}_{2}$ makes it possible to increase the diameter of the pores and develop a narrow distribution of the size of the pores [136]. This improves the microporosity of the activated carbon and increases the adsorption for large organic molecules. The results showed that MB adsorbs better than phenol with adsorption capacities of 390 and $211 \mathrm{mg} / \mathrm{g}$, respectively [136].

Activated carbon obtained from date palm petiole via chemical activation using sodium hydroxide showed a surface area of $655 \mathrm{~m}^{2} / \mathrm{g}$, with a mesoporous structure [135]. This mesoporosity 
is a favorable structural property for use in the elimination of indigo carmine from aqueous solution in batch and continuous column systems. In batch mode, the adsorption was chemical and exothermic, and it underwent pseudo-second-order model kinetics. The adsorption mechanism is related to the physisorption enhanced by chemisorption. The adsorption capacity of the indigo carmine decreases from 53.76 to $32.57 \mathrm{mg} / \mathrm{g}$ if the temperature rises to $40^{\circ} \mathrm{C}$ [135]. However, in continuous mode, this activated carbon provides good efficiency removal of indigo carmine after three repetition cycles [135]. It appears that the adsorption is largely influenced by the $p H$ of the solution and the $\mathrm{pH}_{\mathrm{ZCN}}$. The effect of $p H$ on adsorption can manifest itself through two mechanisms: an electrostatic interaction between the functional groups of the adsorbent (carboxylic, phenolic, lactonic, basic functions, etc.) and the adsorbate, or a chemical reaction between adsorbent and adsorbate. In fact, at $p H<p H_{\mathrm{ZCN}}$, the surface of the carbon is positively charged, which promotes the adsorption of the anions, while the adsorption of cations is favored at $p H>p H_{\mathrm{ZCN}}$, where the surface of the carbon is negatively charged [28-30,135,159,176,184,196,211,222-224]. Tu et al. [183] studied the sorption properties of Bermuda grass derived activated carbon for $\mathrm{Cr}(\mathrm{VI})$ removal. Sorption of $\mathrm{Cr}(\mathrm{VI})$ ions depends on $p H$ of the solution; the best $\mathrm{Cr}(\mathrm{VI})$ uptake was reported at $p H 2.0$ with a maximum adsorption capacity of $403.2 \mathrm{mg} / \mathrm{g}$, and the removal mechanism was predominantly chemisorption. The same observations were reported by Bedada et al. [207] using activated carbon produced from Parthenium hysterophorus weed chemically activated by phosphoric acid. This is to highlight that mostly metal ions $\left(\mathrm{Pb}^{2+}, \mathrm{Cd}^{2+}, \mathrm{Cr}^{3+}, \mathrm{Fe}^{2+}, \mathrm{Cu}^{2+}, \mathrm{Ni}^{2+}, \mathrm{Co}^{2+}, \mathrm{Hg}^{2+}\right.$, etc.) removal is favorable in the $p H$ range 2-6 [143,163,188,192,213,219].

In addition, the search for inexpensive alternative resources as well as methods of preparing activated carbon has attracted the attention of scientific researchers. Several researchers are interested in converting waste from the polymer and textile industries into activated carbon as a favorable approach [176,206,217-219,225]. In this context, activated carbon derived from acrylic fiber waste is obtained by physical activation in the presence of air via controlled heat treatment and used as an adsorbent for the removal of MB from aqueous media [176]. The results indicate that the adsorption of $\mathrm{MB}$ on activated carbon requires a longer equilibrium time when the initial concentration of dye is increased with an adsorption capacity of $8.7 \mathrm{mg} / \mathrm{g}$ [176]. Likewise, Hassan and Elhadidy transformed carpet waste into activated carbon by activation with $\mathrm{H}_{3} \mathrm{PO}_{4}$ at different impregnation ratios [218]. They found that the specific surface area of activated carbon increases with the impregnation ratio, which reaches $953 \mathrm{~m}^{2} / \mathrm{g}$ for an impregnation ratio of 3 . This carbon has a mesoporous structure promoting the adsorption of MB where the capacity of adsorption is $769.2 \mathrm{mg} / \mathrm{g}$ [218]. A study of the effects of the impregnation rate, the carbonization temperature, and the time on the characteristics of the active carbon produced from tomato waste by chemical activation with $\mathrm{ZnCl}_{2}$, was carried out by Saygili and Güzel [216]. This study showed that the influence of the operating parameters on the properties of the pores of the carbons followed a sequence of carbonization temperature> carbonization time $>$ impregnation rate. The activated carbon produced under optimal conditions has a specific surface of $1093 \mathrm{~m}^{2} / \mathrm{g}$ with a mesoporous structure. This carbon has been used effectively for the adsorption of large MB and metanil yellow (MY) molecules, showing maximum monolayer adsorption capacities of $400 \mathrm{mg} / \mathrm{g}$ for MB and $385 \mathrm{mg} / \mathrm{g}$ for MY [216]. The same observations were made by Wang et al. [167] during the preparation of carbon derived from peanut shell by activation by potassium hydroxide.

Certain inorganic anions constitute a group of pollutants in wastewater that are known by their toxic and carcinogenic effects. Among these ions are the nitrates $\left(\mathrm{NO}^{3-}\right)$ and fluoride $\left(\mathrm{F}^{-}\right)$ions. It was reported that activated charcoal, derived from Rice husk developed by chemical activation using potassium carbonate as an activating agent followed by modification by urea, could remove the nitrate ions from aqueous solutions with a maximum adsorption capacity of $8.1 \mathrm{mg} / \mathrm{g}$ [144]. In addition, activated carbon has been used effectively for defluorination of water. By way of example, the citrus limetta peels give, by activation with $\mathrm{FeCl}_{3}$, an activated carbon with a maximum monolayer 
capacity of $9.7 \mathrm{mg} / \mathrm{g}$ under the optimal adsorbent dosage conditions $1 \mathrm{~g} / \mathrm{L}$, a contact time of $240 \mathrm{~min}$, and a temperature of $25^{\circ} \mathrm{C}$ with fluoride concentration ranging from 5 to $30 \mathrm{mg} / \mathrm{L} \mathrm{[28]}$.

It should be noted that an understanding of the activation process is a fundamental step for optimizing the preparation of activated carbon. Indeed, various chemical, physical, and physicochemical processes are used for the synthesis of activated carbon from different natural or synthetic resources. Chemical treatment involves the use of activating agents by impregnation followed by pyrolysis, whereas the physical process involves a high temperature heat treatment in the presence of vapor or carbon dioxide. However, the combination of these two methods has also been used for the production of activated carbon. It remains difficult to predict the physicochemical and morphological properties of carbons according to the treatment applied. It seems that the morphological, the physicochemical, the textural, and the surface properties of activated carbon change significantly by changing the activation time and temperature and the nature of the precursor used. The chemical nature of the surface of activated carbon depends on the type of heteroatoms, such as oxygen, nitrogen, sulfur, etc., that are present on the surface. These hetero atoms or groups accumulated on the surface either come from the starting material itself, or are incorporated during the activation process. This is largely related to the nature of the precursor. All these properties are of great importance for the use of carbons as an adsorbent of pollutants in aqueous solutions. Indeed, the adsorption of pollutants onto activated carbon is a complicated process, and various interactions can coexist during the process involving physical actions and/or chemical bonds. Thus, the adsorption mechanism referred to as electrostatic interactions, hydrogen bonds, $\pi-\pi$ stacking interactions, pore filling, hydrophobic interactions, ion exchange, and also other interactions (n- $\pi$ interactions, etc.) and complexation reactions can take place $[30,184,187,194,197,203,205,220,226-228]$. Figure 5 illustrates the principal interaction methods for adsorption of pollutants on activated carbon.

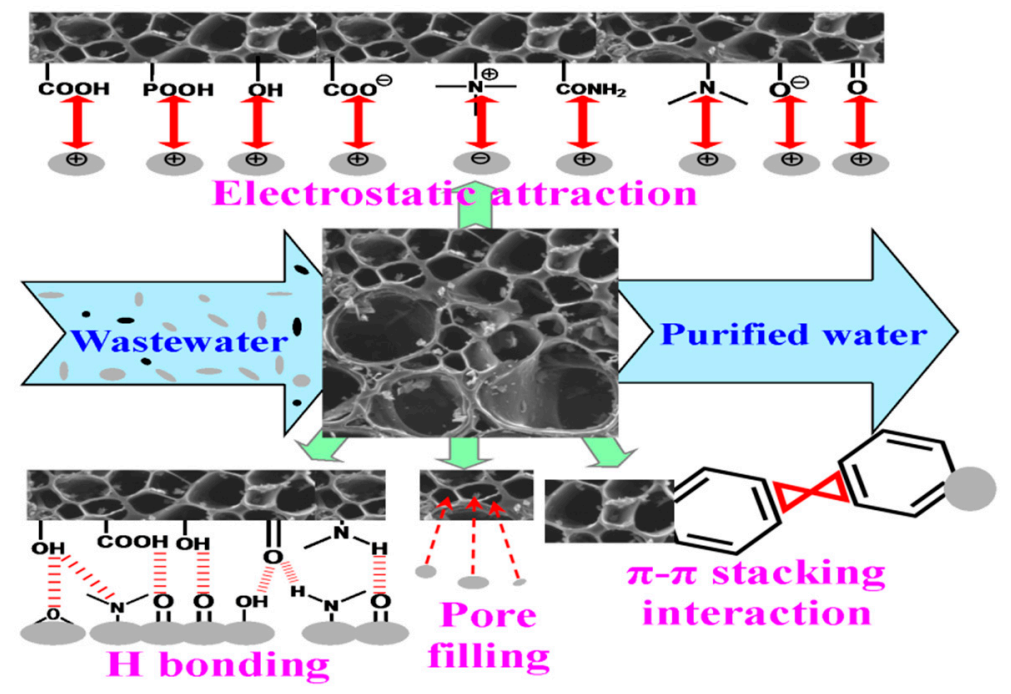

Figure 5. Postulated mechanisms of the interactions of pollutant with activated carbon.

The micropores and mesopores of the porous network of activated carbon contribute to the adsorption of pollutants by pore-filling mechanisms according to their total volume and the size of the pollutant molecules, and this is influenced by the affinity of the adsorbent with respect to the pollutant [229]. The $\pi-\pi$ stacking interactions are suggested for the sorption of aromatic compounds on the surface of activated carbon containing aromatic rings, suggesting the presence of graphene in the carbon [205]. Electrostatic interactions are encountered during the adsorption of ionic and ionizable compounds $[30,135,230]$. Positively charged compounds will tend to sorb onto negatively charged carbon surfaces, while anionic compounds will bind to positively charged sites. These interactions also involve the weak interaction forces and hydrogen bonds between the functional groups (carboxylic, lactonic, phenolic, etc.) present on the surface of the activated carbon and the functional groups of the adsorbate. In fact, the polar groups on the surface of the carbon facilitate the sorption of 
water and promote the formation of hydrogen bonds between the carbon and the sorbates containing electronegative elements $[30,135,230,231]$. Furthermore, the ion exchange and complexation processes are induced by the presence of cations or metal ions in the structure of the activated carbon $[226,230,232]$.

\section{Conclusions}

Carbon materials are one of the most studied areas in the scientific community. Indeed, these materials have remarkable physical and chemical properties, and this explains their uses in various fields such as biology, chemistry of materials, energy storage, electrochemistry, and wastewater treatment. In addition, despite several advances made, researchers face many challenges concerning the synthesis, morphological uniformity, and reproducibility of carbonaceous materials. Graphene and graphite offer excellent electrical mobility that favors their use for the photodegradation of organic pollutants and the manufacture of anodes for lithium-ion batteries and pollution sensors. Thus, materials derived from graphene offer greater flexibility in modifying the properties of the catalyst according to specific requirements. In this respect, the morphological properties of carbon nanotubes combined with their chemical and thermal conduction properties give them an interesting future as a catalyst support. In the same context, activated carbon is widely used in the field of water treatment because of their adsorption properties that result from the diversity of chemical, structural, and textural composition. These properties are related to the precursors and the method of preparation of the activated carbon. In this review, the performance of the retention of pollutants from wastewater was detailed on the basis of recent examples from the literature. It turns out that mastering the methods of preparing carbonaceous materials for larger-scale production and understanding the many properties of these materials are important steps to be taken in order to be able to assist with their applications in the various areas of interest.

Author Contributions: M.b.M. and L.M. designed the manuscript and wrote the main text. R.K. and Y.M. designed and revised the manuscript. All authors have read and agreed to the published version of the manuscript.

Funding: This research received no external funding.

Acknowledgments: The authors would like to express their deep gratitude to Mohamed Naceur Belgacem, the director of Grenoble INP-Pagora and Agefpi, for his valuable advice and assistance, and also to the Tunisian Ministry of Higher Education for its financial support.

Conflicts of Interest: The authors declare no conflict of interest.

$\begin{array}{ll}\text { Abbreviations } & \\ \text { 2,4-DNP } & \text { 2,4-Dinitrophenol } \\ \text { 2,4-DNT-3-SO3 } & \text { 2,4-dinitrotoluene-3-sulfonate } \\ \text { 2,4-DNT-5-SO3 } & \text { 2,4-dinitrotoluene-5-sulfonate } \\ \text { 2,4,6-TNP } & \text { 2,4,6-Trinitrphenol } \\ \text { 4-ClP } & \text { 4-Chlorophenol } \\ \text { 4-NP } & \text { 4-nitrophenol } \\ \text { Activ. Temp. } & \text { Activation temperature } \\ \text { Activ. Time } & \text { Activation time } \\ \text { AMX } & \text { Amoxicillin } \\ \text { Bis-A } & \text { Bisphenol A } \\ \mathrm{C}_{0} & \text { Concentration initial } \\ \text { FBA } & \text { Fixed bed adsorption } \\ \text { MB } & \text { Methylene blue } \\ \text { Me-Red } & \text { Methyl red } \\ \text { MG } & \text { Malachite green } \\ \text { MO } & \text { Methyl orange } \\ \text { Ms } & \text { Municipal sludge } \\ \text { MW } & \text { Microwave }\end{array}$




$\begin{array}{ll}\text { MWCNTs } & \text { Multiwalled carbon nanotubes } \\ \text { MY } & \text { Metanil yellow } \\ \text { PAN } & \text { Polyacrylonitrile } \\ \text { PET } & \text { Polyethyleneterephthalate } \\ p H_{\text {ZCN }} & p H \text { of the point of zero charge } \\ \text { Q }_{\max } & \text { Maximum adsorption capacity } \\ \text { RB5 } & \text { Reactive Black 5 } \\ \text { Rh-B } & \text { Rhodamine B } \\ S_{\text {BET }} & \text { Brunauer-Emmett-Teller area } \\ \text { SDS } & \text { Sodium dodecylbenzene sulfonate } \\ \text { SWCNTs } & \text { Single-walled carbon nanotubes } \\ \text { TMA } & \text { TriMethylAmine }\end{array}$

\section{References}

1. Rouquerol, F.; Luciani, L.; Llewellyn, P.; Denoyel, R.; Rouquerol, J. Techniques de L'ingénieur, Analyse et Caractérisation: Texture des Matériaux Pulvérulents ou Poreux; Inist-CNRS-Paris: Paris, France, 2003; pp. 1050-1057.

2. Besson, J.; Billon, N.; Cantournet, S.; Chastel, Y.; Lorenzon, A.F.G.; Haudin, J.M.; Monasse, B.; Naze, L. Matériaux pour l'ingénieur; Edition Mines: Paris, France, 2006; pp. 81-82.

3. Ma, T.Y.; Yuan, Z.Y. Bioinspired Approach to Synthesizing Hierarchical Porous Materials, Hierarchically Structured Porous Materials: From Nanoscience to Catalysis, Separation, Optics, Energy, and Life Science, 1st ed.; Su, B.L., Sanchez, C., Yang, X.Y., Eds.; Wiley: Weinheim, Germany, 2012; pp. 173-207.

4. Zhuo, H.; Hu, Y.; Tong, X.; Zhong, L.; Peng, X.; Sun, R. Sustainable hierarchical porous carbon aerogel from cellulose for high performance supercapacitor and $\mathrm{CO}_{2}$ capture. Ind. Crop. Prod. 2016, 87, 229-235. [CrossRef]

5. Canal-Rodríguez, M.; Menéndez, J.A.; Arenillas, A. Performance of carbon xerogel-graphene hybrids as electrodes in aqueous supercapacitors. Electrochim. Acta 2018, 276, 28-36.

6. Sarvi, M.N.; Bee, T.B.; Gooi, C.K.; Woonton, B.W.; Gee, M.L.; O'Connor, A.J. Development of functionalized mesoporous silica for adsorption and separation of dairy proteins. Chem. Eng. J. 2014, 235, $244-251$. [CrossRef]

7. Xin, Y.; Peng, S.; Chen, J.; Yang, Z.; Zhang, J. Continuous flow synthesis of porous materials. Chin. Chem. Lett. 2019, 31, 1448-1461. [CrossRef]

8. Wei, Y.; Parmentier, T.E.; de Jong, K.P.; Zecevic, J. Tailoring and visualizing the pore architecture of hierarchical zeolites. Chem. Soc. Rev. 2015, 44,7234-7261. [CrossRef]

9. Zhong, W.; Liu, H.; Bai, C.; Liao, S.; Li, Y. Base-Free Oxidation of Alcohols to Esters at Room Temperature and Atmospheric Conditions using Nanoscale Co-Based Catalysts. ACS Catal. 2015, 5, 1850-1856. [CrossRef]

10. Xu, L.; Guo, L.; Hu, G.; Chen, J.; Hu, X.; Wang, S.; Dai, W.; Fan, M. Nitrogen-doped porous carbon spheres derived from d-glucose as highly-efficient $\mathrm{CO}_{2}$ sorbents. RSC Adv. 2015, 5, 37964-37969. [CrossRef]

11. Li, D.; Chen, Y.; Zheng, M.; Zhao, H.; Zhao, Y.; Sun, Z. Hierarchically structured porous nitrogen-doped carbon for highly selective $\mathrm{CO}_{2}$ capture. ACS Sustain. Chem. Eng. 2016, 4, 298-304. [CrossRef]

12. Xu, A.-R.; Chen, L.; Guo, X.; Xiao, Z.; Liu, R. Biodegradable lignocellulosic porous materials: Fabrication, characterization and its application in water processing. Int. J. Biol. Macromol. 2018, 115, 846-852. [CrossRef]

13. Zhu, L.; Shen, D.; Luo, K.H. A critical review on VOCs adsorption by different porous materials: Species, mechanisms and modification methods. J. Hazard. Mater. 2020, 389, 122102. [CrossRef]

14. Yang, F.; Sun, L.; Zhang, W.; Zhang, Y. One-pot synthesis of porous carbon foam derived from corn straw: Atrazine adsorption equilibrium and kinetics. Environ. Sci. Nano. 2017, 4, 625-635. [CrossRef]

15. Little, I.; Alorkpa, E.; Khan, V.; Povazhnyi, V.; Vasiliev, A. Efficient porous adsorbent for removal of cesium from contaminated water. J. Porous Mater. 2019, 26, 361-369. [CrossRef]

16. Zhu, Y.; Hu, H.; Li, W.; Zhang, X. Resorcinol-formaldehyde based porous carbon as an electrode material for supercapacitors. Carbon 2007, 45, 160-165. [CrossRef]

17. Zhang, W.F.; Huang, Z.-H.; Cao, G.P.; Kang, F.; Yang, Y. A novel mesoporous carbon with straight tunnel-like pore structure for high rate electrochemical capacitors. J. Power Sources 2012, 204, 230-235. [CrossRef] 
18. Wu, K.; Liu, Q. Nitrogen-doped mesoporous carbons for high performance supercapacitors. Appl. Surf. Sci. 2016, 379, 132-139. [CrossRef]

19. Wang, P.; Xu, J.; Xu, F.; Zhao, W.; Sun, P.; Zhang, Z.; Qian, M.; Huang, F. Constructing hierarchical porous carbon via tin punching for efficient electrochemical energy storage. Carbon 2018, 134, 391-397. [CrossRef]

20. Wang, Y.; Qu, Q.; Gao, S.; Tang, G.; Liu, K.; He, S.; Huang, C. Biomass derived carbon as binder-free electrode materials for supercapacitors. Carbon 2019, 155, 706-726. [CrossRef]

21. Yu, K.; Wang, J.; Wang, X.; Liang, J.; Liang, C. Sustainable application of biomass by-products: Corn straw-derived porous carbon nanospheres using as anode materials for lithium ion batteries. Mater. Chem. Phys. 2020, 243, 122644. [CrossRef]

22. Huang, J.; Liang, Y.; Hu, H.; Liu, S.; Cai, Y.; Dong, H.; Zheng, M.; Xiao, Y.; Liu, Y. Ultrahigh-surface-area hierarchical porous carbon from chitosan: Acetic acid mediated efficient synthesis and its application in superior supercapacitors. J. Mater. Chem. 2017, 5, 24775-24781. [CrossRef]

23. Chen, Z.; Ye, S.; Evans, S.D.; Ge, Y.; Zhu, Z.; Tu, Y.; Yang, X. Confined assembly of hollow carbon spheres in carbonaceous nanotube: A spheres-in-tube carbon nanostructure with hierarchical porosity for high-performance supercapacitor. Small 2018, 14, 1704015. [CrossRef]

24. Kim, B.; Park, J.; Baik, S.; Lee, J.W. Spent coffee derived hierarchical porous carbon and its application for energy storage. J. Porous Mater. 2020, 27, 451-463. [CrossRef]

25. Marsh, H.; Rodriguez-Reinoso, F. Activated Carbon; Elsevier: Amsterdam, The Netherlands, 2006.

26. Yang, Y.; Wang, H.; Yan, F.-Y.; Qi, Y.; Lai, Y.-K.; Zeng, D.-M.; Chen, G.; Zhang, K.-Q. Bioinspired Porous Octacalcium Phosphate/Silk Fibroin Composite Coating Materials Prepared by Electrochemical Deposition. ACS Appl. Mater. Interfaces 2015, 7, 5634-5642. [CrossRef] [PubMed]

27. Pallares, J.; Cencerrado, A.G.; Arauzo, I. Production and characterization of activated carbon from barley straw by physical activation with carbon dioxide and steam. Biomass Bioenergy 2018, 115, 64-73. [CrossRef]

28. Siddique, A.; Nayak, A.K.; Singh, J. Synthesis of FeCl3-activated carbon derived from waste Citrus limetta peels for removal of fluoride: An eco-friendly approach for the treatment of groundwater and bio-waste collectively. Groundw. Sustain. Dev. 2020, 10, 100339. [CrossRef]

29. Chiang, C.-H.; Chen, J.; Lin, J.-H. Preparation of pore-size tunable activated carbon derived from waste coffee grounds for high adsorption capacities of organic dyes. J. Environ. Chem. Eng. 2020, 8, 103929. [CrossRef]

30. Giraldo, S.; Robles, I.; Ramirez, A.; Florez, E.; Acelas, N. Mercury removal from wastewater using agroindustrial waste adsorbents. SN Appl. Sci. 2020, 2, 1029. [CrossRef]

31. Danish, M.; Ahmad, T. A review on utilization of wood biomass as a sustainable precursor for activated carbon production and application. Renew. Sustain. Energy Rev. 2018, 87, 1-21. [CrossRef]

32. Wong, S.; Ngadi, N.; Inuwa, I.M.; Hassan, O. Recent advances in applications of activated carbon from biowaste for wastewater treatment: A short review. J. Clean. Prod. 2018, 175, 361-375. [CrossRef]

33. Sing, K.S.W. Reporting physisorption data for gas/solid systems with special reference to the determination of surface area and porosity (Recommendations 1984). Pure Appl. Chem. 1985, 57, 603-619. [CrossRef]

34. Thommes, M.; Kaneko, K.; Neimark, A.V.; Olivier, J.P.; Rodriguez-Reinoso, F.; Rouquerol, J.; Sing, K.S.W. Physisorption of gases, with special reference to the evaluation of surface area and pore size distribution (IUPAC Technical Report). Pure Appl. Chem. 2015, 87, 1051-1069. [CrossRef]

35. Mnasri, N.; Charnay, C.; Ménorval, L.C.; Moussaoui, Y.; Elaloui, E.; Zajac, J. Silver nanoparticle-containing submicron-in-size mesoporous silica-based systems for iodine entrapment and immobilization from gas phase. Microporous Mesoporous Mater. 2014, 196, 305-313. [CrossRef]

36. Costa, J.A.S.; Jesus, R.A.; Santos, D.; Manoa, J.F.; Romao, L.P.C.; Paranhos, C.M. Recent progresses in the adsorption of organic, inorganic, and gas compounds by MCM-41-based mesoporous materials. Microporous Mesoporous Mater. 2020, 291, 109698. [CrossRef]

37. Luo, X.; Guoa, J.; Chang, P.; Qian, H.; Pei, F.; Wang, W.; Miao, K.; Guo, S.; Feng, G. ZSM-5@MCM-41 composite porous materials with a core-shell structure: Adjustment of mesoporous orientation basing on interfacial electrostatic interactions and their application in selective aromatics transport. Sep. Purif. Technol. 2020, 239, 116516. [CrossRef]

38. Zhang, C.; Zhao, P.; Liu, S.; Yu, K. Three-dimensionally ordered macroporous perovskite materials for environmental applications. Chin. J. Catal. 2019, 40, 1324-1338. [CrossRef] 
39. Zeng, F.; Zhang, X.; Pan, Y.; Xu, M.; Yang, L.; Qu, Y.; Guo, M.; Yuan, C. Graphene-templated growth of vertical $\mathrm{MnO}$ nanosheets with open macroporous architectures as anode materials for fast lithium storage. J. Alloys Compd. 2018, 769, 10-17. [CrossRef]

40. Simaioforidou, A.; Kostas, V.; Karakassides, M.A.; Louloudi, M. Surface chemical modification of macroporous and mesoporous carbon materials: Effect on their textural and catalytic properties. Microporous Mesoporous Mater. 2019, 279, 334-344. [CrossRef]

41. Lal, S.; Prat, M.; Plamondon, M.; Poulikakos, L.; Partl, M.N.; Derome, D.; Carmeliet, J. A cluster-based pore network model of drying with corner liquid films, with application to a macroporous material. Int. J. Heat Mass Transf. 2019, 140, 620-633. [CrossRef]

42. Deprez, N.; McLachlan, D.S. The analysis of the electrical conductivity of graphite conductivity of graphite powders during compaction. J. Phys. D Appl. Phys. 1988, 21, 101-107. [CrossRef]

43. Liu, Y.; Li, X.; Fan, L.; Li, S.; Sari, H.M.K.; Qin, J. A Review of Carbon-Based Materials for Safe Lithium Metal Anodes. Front. Chem. 2019, 7, 721. [CrossRef]

44. Marinho, B.; Ghislandi, M.; Koning, C.E.; de With, G. Electrical conductivity of compacts of graphene, multi-wall carbon nanotubes, carbon black, and graphite powder. Powder Technol. 2012, 221, 351-358. [CrossRef]

45. Jana, P.; Fierro, V.; Pizzi, A.; Celzard, A. Thermal conductivity improvement of composite carbon foams based on tannin-based disordered carbon matrix and graphite fillers. Mater. Des. 2015, 83, 635-643. [CrossRef]

46. Dong, Y.; Meng, M.; Groves, M.M.; Zhang, C.; Lin, J. Thermal conductivities of two-dimensional graphitic carbon nitrides by molecule dynamics simulation. Int. J. Heat Mass Transf. 2018, 123, 738-746. [CrossRef]

47. Duan, J.; Wu, W.; Nolan, A.M.; Wang, T.; Wen, J.; Hu, C.; Mo, Y.; Luo, W.; Huang, Y. Lithium graphite paste: An interface compatible anode for solid-state batteries. Adv. Mater. 2019, 31, 1807243. [CrossRef] [PubMed]

48. Luo, H.-M.; Chen, H.; Chen, Y.-Z.; Li, P.; Zhang, J.-Q.; Zhao, X. Simple synthesis of porous carbon materials for high-performance supercapacitors. J. Appl. Electrochem. 2016, 46, 703-712. [CrossRef]

49. Li, G.; Yin, Z.; Guo, H.; Wang, Z.; Yan, G.; Yang, Z.; Liu, Y.; Ji, X.; Wang, J. Metalorganic Quantum Dots and Their Graphene-Like Derivative Porous Graphitic Carbon for Advanced Lithium-Ion Hybrid Supercapacitor. Adv. Energy Mater. 2019, 9, 1802878. [CrossRef]

50. Li, G.; Yang, Z.; Yin, Z.; Guo, H.; Wang, Z.; Yan, G.; Liu, Y.; Li, L.; Wang, J. Non-aqueous dual-carbon lithium-ion capacitors: A review. J. Mater. Chem. A 2019, 7, 15541-15563. [CrossRef]

51. Zhong, Y.; Guo, Q.; Li, S.; Shi, J.; Liu, L. Heat transfer enhancement of paraffin wax using graphite foam for thermal energy storage. Sol. Energy Mater. Sol. Cells 2010, 94, 1011-1014. [CrossRef]

52. Zhang, J.; Xu, T.; Cong, Y.; Zhang, Y.; Li, X.; Dong, Z.; Li, Y.; Yuan, G.; Zhang, J.; Cui, Z. Improved rate performance and cycling stability of graphitized mesoporous carbon as anode materials for lithium-ion batteries. J. Mater. Sci. 2019, 54, 648-658. [CrossRef]

53. Pitchaiya, S.; Eswaramoorthy, N.; Natarajan, M.; Santhanam, A.; Asokan, V.; Ramakrishnan, V.M.; Rangasamy, B.; Sundaram, S.; Ravirajan, P.; Velauthapillai, D. Perovskite Solar Cells: A Porous Graphitic Carbon based Hole Transporter/Counter Electrode Material Extracted from an Invasive Plant Species Eichhornia Crassipes. Sci. Rep. 2020, 10, 6835. [CrossRef]

54. Horiuchi, S.; Gotou, T.; Fujiwara, M.; Asaka, T.; Yokosawa, T.; Matsui, Y. Single graphene sheet detected in a carbon nanofilm. Appl. Phys. Lett. 2004, 84, 2403-2405. [CrossRef]

55. Novoselov, K.S.; Geim, A.K.; Morozov, S.V.; Jiang, D.; Zhang, Y.; Dubonos, S.V.; Grigorieva, I.V.; Firsov, A.A. Electric Field Effect in Atomically Thin Carbon Films. Science 2004, 306, 666-669. [CrossRef] [PubMed]

56. Berger, C.; Song, Z.; Li, T.; Li, X.; Ogbazghi, A.Y.; Feng, R.; Dai, Z.; Marchenkov, A.N.; Conrad, E.H.; First, P.N.; et al. Ultrathin Epitaxial Graphite: 2D Electron Gas Properties and a Route toward Graphene-based Nanoelectronics. J. Phys. Chem. B 2004, 108, 19912-19916. [CrossRef]

57. Novoselov, K.S.; Geim, A.K.; Morozov, S.V.; Jiang, D.; Katsnelson, M.I.; Grigorieva, I.V.; Dubonos, S.V.; Firsov, A.A. Two-dimensional gas of massless Dirac fermions in graphene. Nature 2005, 438, 197-200. [CrossRef] [PubMed]

58. Allen, M.J.; Tung, V.C.; Kaner, R.B. Honeycomb Carbon: A Review of Graphene. Chem. Rev. 2010, 110, 132-145. [CrossRef]

59. Nair, R.R.; Blake, P.; Grigorenko, A.N.; Novoselov, K.S.; Booth, T.J.; Stauber, T.; Peres, N.M.R.; Geim, A.K. Fine Structure Constant Defines Visual Transparency of Graphene. Science 2008, 320, 1308. [CrossRef] 
60. Varghese, S.S.; Lonkar, S.; Singh, K.K.; Swaminathan, S.; Abdala, A. Recent advances in graphene based gas sensors. Sens. Actuators B Chem. 2015, 218, 160-183. [CrossRef]

61. You, P.; Liu, Z.; Tai, Q.; Liu, S.; Yan, F. Efficient Semitransparent Perovskite Solar Cells with Graphene Electrodes. Adv. Mater. 2015, 27, 3632-3638. [CrossRef]

62. De Nicola, F.; Purayil, N.S.P.; Miŝeikis, V.; Spirito, D.; Tomadin, A.; Coletti, C.; Polini, M.; Krahne, R.; Pellegrini, V. Graphene Plasmonic Fractal Metamaterials for Broadband Photodetectors. Sci. Rep. 2020, 10, 6882. [CrossRef]

63. Emtsev, K.V.; Bostwick, A.; Horn, K.; Jobst, J.; Kellogg, G.L.; Ley, L.; McChesney, J.L.; Ohta, T.; Reshanov, S.A.; Röhrl, J.; et al. Towards wafer-size graphene layers by atmospheric pressure graphitization of silicon carbide. Nat. Mater. 2009, 8, 203-207. [CrossRef]

64. Guo, Z.; Dong, R.; Chakraborty, P.S.; Lourenco, N.; Palmer, J.; Hu, Y.; Ruan, M.; Hankinson, J.; Kunc, J.; Cressler, J.D.; et al. Record maximum oscillation frequency in C-face epitaxial graphene transistors. Nano Lett. 2013, 13, 942-947. [CrossRef]

65. Tetlow, H.; Posthuma de Boer, J.; Ford, I.J.; Vvedensky, D.D.; Coraux, J.; et Kanto rovich, L. Growth of epitaxial graphene: Theory and experiment. Phys. Rep. 2014, 542, 195-295. [CrossRef]

66. Eda, G.; Fanchini, G.; Chhowalla, M. Large-area ultrathin films of reduced graphene oxide as a transparent and flexible electronic material. Nat. Nanotechnol. 2008, 3, 270-274. [CrossRef] [PubMed]

67. Ago, H.; Ogawa, Y.; Tsuji, M.; Mizuno, S.; Hibino, H. Catalytic growth of graphene: Toward large-area single-crystalline graphene. J. Phys. Chem. Lett. 2012, 3, 2228-2236. [CrossRef] [PubMed]

68. Mattevi, C.; Kim, H.; Chhowalla, M. A review of chemical vapour deposition of graphene on copper. J. Mater. Chem. 2011, 21, 3324-3334. [CrossRef]

69. Rabchinskii, M.K.; Ryzhkov, S.A.; Kirilenko, D.A.; Ulin, N.V.; Baidakova, M.V.; Shnitov, V.V.; Pavlov, S.I.; Chumakov, R.G.; Stolyarova, D.Y.; Besedina, N.A.; et al. From graphene oxide towards aminated graphene: Facile synthesis, its structure and electronic properties. Sci. Rep. 2020, 10, 6902. [CrossRef] [PubMed]

70. Mayorov, A.S.; Gorbachev, R.V.; Morozov, S.V.; Britnell, L.; Jalil, R.; Ponomarenko, L.A.; Blake, P.; Novoselov, K.S.; Watanabe, K.; Taniguchi, T.; et al. Micrometer-scale ballistic transport in encapsulated graphene at room temperature. Nano Lett. 2011, 11, 2396-2399. [CrossRef] [PubMed]

71. Soldano, C.; Mahmood, A.; Dujardin, E. Production, properties and potential of grapheme. Carbon 2010, 48, 2127-2150. [CrossRef]

72. Kang, H.-K.; Woo, S.-G.; Kim, J.-H.; Lee, S.-R.; Lee, D.-G.; and Yu, J.-S. Three-dimensional monolithic corrugated graphene/Ni foam for highly stable and efficient Li metal electrode. J. Power Sources 2019, 413, 467-475. [CrossRef]

73. Liu, W.; Xia, Y.; Wang, W.; Wang, Y.; Jin, J.; Chen, Y.; Paek, E.; Mitlin, D. Pristine or highly defective? Understanding the role of graphene structure for stable lithium metal plating. Adv. Energy Mater. 2019, 9, 1802918. [CrossRef]

74. Kang, H.K.; Woo, S.G.; Kim, J.H.; Yu, J.S.; Lee, S.R.; Kim, Y.J. Fewlayer graphene island seeding for dendrite-free Li metal electrodes. ACS Appl. Mater. Interfaces 2016, 8, 26895-26901. [CrossRef]

75. Lin, D.; Liu, Y.; Liang, Z.; Lee, H.W.; Sun, J.; Wang, H.; Yan, K.; Xie, J.; Cui, Y. Layered reduced graphene oxide with nanoscale interlayer gaps as a stable host for lithium metal anodes. Nat. Nanotechnol. 2016, 11, 626-632. [CrossRef] [PubMed]

76. Suvarnaphaet, P.; Pechprasarn, S. Graphene-Based Materials for Biosensors: A Review. Sensors 2017, $17,2161$. [CrossRef] [PubMed]

77. Kang, M.-A.; Ji, S.; Kim, S.; Park, C.-Y.; Myung, S.; Song, W.; Lee, S.S.; Lima, J.; An, K.-S. Highly sensitive and wearable gas sensors consisting of chemically functionalized graphene oxide assembled oncotton yarn. RSC Adv. 2018, 8, 11991-11996. [CrossRef]

78. Hong, H.S.; Phuong, N.H.; Huong, N.T.; Nam, N.H.; Hue, N.T. Highly sensitive and low detection limit of resistive $\mathrm{NO}_{2}$ gas sensor based on a $\mathrm{MoS}_{2}$ /graphene two-dimensional heterostructures. Appl. Surf. Sci. 2019, 492, 449-454. [CrossRef]

79. Chou, C.-Y.; Tseng, S.-F.; Chang, T.-L.; Tu, C.-T.; Han, H.-C. Controlled bridge growth of ZnO nanowires on laser-scribed graphene-based devices for NO gas detection. Appl. Surf. Sci. 2020, 508, 145204. [CrossRef]

80. Hoang, N.V.; Hung, C.M.; Hoa, N.D.; Duy, N.V.; Park, I.; Hieu, N.V. Excellent detection of $\mathrm{H}_{2} \mathrm{~S}$ gas at ppb concentrations using $\mathrm{ZnFe}_{2} \mathrm{O}_{4}$ nanofibers loaded with reduced graphene oxide. Sens. Actuators B: Chem. 2019, 282, 876-884. [CrossRef] 
81. Pearce, R.; Iakimov, T.; Andersson, M.; Hultman, L.; Spetz, A.L.; Yakimova, R. Epitaxially grown graphene based gas sensors for ultra sensitive $\mathrm{NO}_{2}$ detection. Sens. Actuators B Chem. 2011, 155, 451-455. [CrossRef]

82. Shaban, M.; Ali, S.; Rabiaj, M. Design and application of nanoporous graphene oxide film for $\mathrm{CO}_{2}, \mathrm{H}_{2}$, and $\mathrm{C}_{2} \mathrm{H}_{2}$ gases sensing. J. Mater. Res. Technol. 2019, 8, 4510-4520. [CrossRef]

83. Fan, Y.-Y.; Tu, H.-L.; Pang, Y.; Wei, F.; Zhao, H.-B.; Yang, Y.; Ren, T.-L. Au-decorated porous structure graphene with enhanced sensing performance for low-concentration $\mathrm{NO}_{2}$ detection. Rare Met. 2020, 39, 651-658. [CrossRef]

84. Alfano, B.; Massera, E.; Miglietta, M.L.; Polichetti, T.; Schiattarella, C.; Di Francia, G. Graphene Decoration for Gas Detection. In Sensors and Microsystems; Leone, A., Forleo, A., Francioso, L., Capone, S., Siciliano, P., Di Natale, C., Eds.; AISEM 2017; Lecture Notes in Electrical Engineering: Springer, 2018; Volume 457, pp. 35-40.

85. Chen, Y.; Gao, B.; Zhao, J.-X.; Cai, Q.-H.; Fu, H.-G. Si-doped graphene: An ideal sensor for NO- or $\mathrm{NO}_{2}$-detectionand metal-free catalyst for $\mathrm{N}_{2} \mathrm{O}$-reduction. J. Mol. Model. 2012, 18, 2043-2054. [CrossRef]

86. Wang, Y.; Li, Y.; Tang, L.; Lu, J.; Li, J. Application of graphene-modified electrode for selective detection of dopamine. Electrochem. Commun. 2009, 11, 889-892. [CrossRef]

87. Brownson, D.A.C.; Foster, C.W.; Banks, C.E. The electrochemical performance of graphene modified electrodes: An analytical perspective. Analyst 2012, 137, 1815-1823. [CrossRef] [PubMed]

88. Lin, W.-J.; Liao, C.-S.; Jhang, J.-H.; Tsai, Y.-C. Graphene modified basal and edge plane pyrolytic graphite electrodes for electrocatalytic oxidation of hydrogen peroxide and $\beta$-nicotinamide adenine dinucleotide. Electrochem. Commun. 2009, 11, 2153-2156. [CrossRef]

89. Kang, X.; Wang, J.; Wu, H.; Liu, J.; Aksay, I.A.; Lin, Y. A graphene-based electrochemical sensor for sensitive detection of paracetamol. Talanta 2010, 81, 754-759. [CrossRef]

90. Wang, Y.; Zhang, D.; Wu, J. Electrocatalytic oxidation of kojic acid at a reduced graphene sheet modified glassy carbon electrode. J. Electroanal. Chem. 2012, 664, 111-116. [CrossRef]

91. Mohan, H.K.S.V.; Chee, W.K.; Li, Y.; Nayak, S.; Poh, C.L.; Thean, A.V.Y. A highly sensitive graphene oxide based label-free capacitive aptasensor for vanillin detection. Mater. Des. 2020, 186, 108208. [CrossRef]

92. Zhang, J.-W.; Wang, K.-P.; Zhang, X. Fabrication of $\mathrm{SnO}_{2}$ decorated graphene composite material and its application in electrochemical detection of caffeic acid in red wine. Mater. Res. Bul. 2020, 126, 110820. [CrossRef]

93. Zuchowska, A.; Chudy, M.; Dybko, A.; Brzozka, Z. Graphene as a new material in anticancer therapy-in vitro studies. Sens. Actuators B Chem. 2017, 243, 152-165. [CrossRef]

94. Cheng, C.; Li, S.; Thomas, A.; Kotov, N.A.; Haag, R. Functional Graphene Nanomaterials Based Architectures: Biointeractions, Fabrications, and Emerging Biological Applications. Chem. Rev. 2017, 117, 1826-1914. [CrossRef]

95. Rajakumar, G.; Zhang, X.-H.; Gomathi, T.; Wang, S.-F.; Ansari, M.A.; Mydhili, G.; Nirmala, G.; Alzohairy, M.A.; Chung, I.-M. Current Use of Carbon-Based Materials for Biomedical Applications-A Prospective and Review. Processes 2020, 8, 355. [CrossRef]

96. Russier, J.; León, V.; Orecchioni, M.; Hirata, E.; Virdis, P.; Fozza, C.; Sgarrella, F.; Cuniberti, G.; Prato, M.; Vázquez, E.; et al. Few-Layer Graphene Kills Selectively Tumor Cells from Myelomonocytic Leukemia Patients. Angew. Chem. Int. Ed. 2017, 56, 3014-3019. [CrossRef] [PubMed]

97. Su, S.; Wang, J.; Vargas, E.; Wei, J.; Martinez-Zaguilan, R.; Sennoune, S.R.; Pantoya, M.L.; Wang, S.; Chaudhuri, J.; Qiu, J. Porphyrin Immobilized Nanographene Oxide for Enhanced and Targeted Photothermal Therapy of Brain Cancer. ACS Biomater. Sci. Eng. 2016, 2, 1357-1366. [CrossRef]

98. Fadlalla, M.I.; Kumar, P.S.; Selvam, V.; Babu, S.G. Emerging energy and environmental applicationof graphene and their composites: A review. J. Mater. Sci. 2020, 55, 7156-7183. [CrossRef]

99. Yao, Y.; Shankar, R.; Rauter, P.; Song, Y.; Kong, J.; Loncar, M.; Capasso, F. High-responsivity mid-infrared graphene detectors with antenna-enhanced photocarrier generation and collection. Nano Lett. 2014, 14, 3749-3754. [CrossRef] [PubMed]

100. Begum, H.; Kim, Y.-B. Improvement of Catalytic Activity of Platinum Nanoparticles Decorated Carbon Graphene Composite on Oxygen Electroreduction for Fuel Cells. Processes 2019, 7, 586. [CrossRef]

101. He, J.; Chen, Y.; Lv, W.; Wen, K.; Li, P.; Wang, Z.; Zhang, W.; Qin, W.; He, W. Three-Dimensional Hierarchical Graphene-CNT@Se: A Highly Efficient Freestanding Cathode for Li-Se Batteries. ACS Energy Lett. 2016, 1, 16-20. [CrossRef] 
102. He, J.; Chen, Y.; Lv, W.; Wen, K.; Xu, C.; Zhang, W.; Qin, W.; He, W. Three-Dimensional CNT/Graphene-Li $2 \mathrm{~S}$ Aerogel as Freestanding Cathode for High-Performance Li-S Batteries. ACS Energy Lett. 2016, 1, 820-826. [CrossRef]

103. He, J.; Chen, Y.; Li, P.; Fu, F.; Wang, Z.; Zhang, W. Three-dimensional CNT/graphene-sulfur hybrid sponges with high sulfur loading as superior capacity cathodes for lithium-sulfur batteries. J. Mater. Chem. A 2015, 3, 18605-18610. [CrossRef]

104. Xu, C.; Jing, Y.; He, J.; Zhou, K.; Chen, Y.; Li, Q.; Lin, J.; Zhang, W. Self-assembled interwoven $\mathrm{CoS}_{2} / \mathrm{CNTs}$ /graphene architecture as anode for high-performance lithium ion batteries. J. Alloys Compd. 2017, 708, 1178-1183. [CrossRef]

105. Iijima, S. Helical microtubules of graphitic carbon. Nature 1991, 354, 56-58. [CrossRef]

106. Bethune, D.S.; Klang, C.H.; De Vries, M.S.; Gorman, G.; Savoy, R.; Vasquez, J.; Beyers, R. Cobalt catalysed growth of carbon nanotubes with single-atomic-layer walls. Nature 1993, 363, 605-607. [CrossRef]

107. Iijima, S.; Ichihashi, T. Single-shell carbon nanotubes of 1-nm diameter. Nature 1993, 363, 603-605. [CrossRef]

108. Inagaki, M. Advanced Carbon Materials. In Handbook of Advanced Ceramics, 2nd ed.; Somiya, S., Ed.; Academic Press: Cambridge, MA, USA, 2013; pp. 25-60.

109. Gupta, S.; Tai, N.H. Carbon materials and their composites for electromagnetic interference shielding effectiveness in X-band. Carbon 2019, 152, 159-187. [CrossRef]

110. Lee, D.W.; Park, J.; Kim, B.J.; Kim, H.; Choi, C.; Baughman, R.H.; Kim, S.J.; Kim, Y.T. Enhancement of electromagnetic interference shielding effectiveness with alignment of spinnable multiwalled carbon nanotubes. Carbon 2019, 142, 528-534. [CrossRef]

111. Kumar, S.; Rani, R.; Dilbaghi, N.; Tankeshwar, K.; Kim, K.-H. Carbon nanotubes: A novel material for multifaceted applications in human healthcare. Chem. Soc. Rev. 2017, 46, 158-196. [CrossRef] [PubMed]

112. Wang, K.; Luo, S.; Wu, Y.; He, X.; Zhao, F.; Wang, J.; Jiang, K.; Fan, S. Super-aligned carbon nanotube films as current collectors for lightweight and flexible lithium ion batteries. Adv. Funct. Mater. 2013, 23, 846-853. [CrossRef]

113. Qi, F.; He, J.; Chen, Y.; Zheng, B.; Li, Q.; Wang, X.; Yu, B.; Lin, J.; Zhou, J.; Li, P.; et al. Few-layered ReS 2 nanosheets grown on carbon nanotubes: A highly efficient anode for high-performance lithium-ion batteries. Chem. Eng. J. 2017, 315, 10-17. [CrossRef]

114. Zhang, R.; Cheng, X.B.; Zhao, C.Z.; Peng, H.J.; Shi, J.L.; Huang, J.Q.; Wang, J.; Wei, F.; Zhang, Q. Conductive nanostructured scaffolds render low local current density to inhibit lithium dendrite growth. Adv. Mater. 2016, 28, 2155-2162. [CrossRef]

115. Deng, Y.; Lu, H.; Cao, Y.; Xu, B.; Hong, Q.; Cai, W.; Yang, W. Multiwalled carbon nanotube interlayers with controllable thicknesses for high capacity and long-life lithium metal anodes. J. Power Sources 2019, 412, 170-179. [CrossRef]

116. Rutherglen, C.; Burke, P. Nanoelectromagnetics: Circuit and Electromagnetic Properties of Carbon Nanotubes. Small 2009, 5, 884-906. [CrossRef]

117. Daneshvar, F.; Aziz, A.; Abdelkader, A.M.; Zhang, T.; Sue, H.-J.; Welland, M.E. Porous $\mathrm{SnO}_{2}-\mathrm{CuxO}$ nanocomposite thin film on carbon nanotubes as electrodes for high performance supercapacitors. Nanotechnology 2018, 30, 015401. [CrossRef] [PubMed]

118. Moreno-Fernandez, G.; Ibanez, J.; Rojo, J.M.; Kunowsky, M. Activated carbon fiber monoliths as supercapacitor electrodes. Adv. Mater. Sci. Eng. 2017,1-8. [CrossRef]

119. Ma, W.L.; Cai, Z.H.; Zhang, Y.; Wang, Z.Y.; Xia, L.; Ma, S.P.; Lia, G.H.; Huang, Y. An Overview of Stretchable Supercapacitors Based on Carbon Nanotube and Graphene. Chin. J. Polym. Sci. 2020, 38, 491-505. [CrossRef]

120. Yang, Z.; Tian, J.; Yin, Z.; Cui, C.; Qian, W.; Wei, F. Carbon nanotube- and graphene-based nanomaterials and applications in high-voltage supercapacitor. Carbon 2019, 141, 467-480. [CrossRef]

121. Duong, H.; Cheng, H.; Jewell, D. Advanced Supercapacitors Using Carbon Nanotubes. Mater. Res. Found. 2017, 12, 207-228.

122. Ying, L.S.; Mohd Salleh, M.A.; Yusoff, H.M.; Rashid, S.B.A.; Razak, J. Continuous production of carbon nanotubes. J. Ind. Eng. Chem. 2011, 17, 367-376. [CrossRef]

123. Tai, N.H.; Chen, H.M.; Chen, Y.J.; Hsieh, P.Y.; Liang, J.R.; Chou, T.W. Optimization of processing parameters of the chemical vapor deposition process for synthesizing high-quality single-walled carbon nanotube fluff and roving. Compos. Sci. Technol. 2012, 72, 1855-1862. [CrossRef] 
124. Guo, T.; Nikolaev, P.; Rinzler, A.G.; Tomanek, D.; Colbert, D.T.; Smalley, R.E. Selfassembly of tubular fullerenes. J. Phys. Chem. 1995, 99, 10694-10697. [CrossRef]

125. Ebbesen, T.W.; Ajayan, P.M. Large-scale synthesis of carbon nanotubes. Nature 1992, 358, 220-222. [CrossRef]

126. Zhou, D.; Anoshkina, E.V.; Chow, L.; Chai, G. Synthesis of carbon nanotubes by electrochemical deposition at room temperature. Carbon 2006, 44, 1013-1016. [CrossRef]

127. Cao, C.; Zhou, Y.; Ubnoske, S.; Zang, J.; Cao, Y.; Henry, P.; Parker, C.B.; Glass, J.T. Highly stretchable supercapacitors via crumpled vertically aligned carbon nanotube forests. Adv. Energy Mater. 2019, 9, 1900618. [CrossRef]

128. Zhang, Z.; Wang, L.; Li, Y.; Wang, Y.; Zhang, J.; Guan, G.; Pan, Z.; Zheng, G.; Peng, H. Nitrogen-doped core-sheath carbon nanotube array for highly stretchable supercapacitor. Adv. Energy Mater. 2017, 7, 1601814. [CrossRef]

129. Hua, C.; Shang, Y.; Wang, Y.; Xu, J.; Zhang, Y.; Li, X.; Cao, A. A flexible gas sensor based on single-walled carbon nanotube- $\mathrm{Fe}_{2} \mathrm{O}_{3}$ composite film. Appl. Surf. Sci. 2017, 405, 405-411. [CrossRef]

130. Tyagi, P.; Sharma, A.; Tomar, M.; Gupta, V. A comparative study of RGO-SnO 2 and $\mathrm{MWCNT}^{-S n O} \mathrm{~S}_{2}$ nanocomposites based $\mathrm{SO}_{2}$ gas sensors, Sens. Actuators B Chem. 2017, 248, 980-986. [CrossRef]

131. Gupta, S.; Murthy, C.N.; Ratna Prabha, C. Recent advances in carbon nanotube based electrochemical biosensors. Int. J. Biol. Macromol. 2018, 108, 687-703. [CrossRef]

132. Farghali, A.A.; Tawab, H.A.A.; Moaty, S.A.A.; Khaled, R. Functionalization of acidified multi-walled carbon nanotubes for removal of heavy metals in aqueous solutions. J. Nanostruct. Chem. 2017, 7, 101-111. [CrossRef]

133. Getachew, T.; Hussen, A.; Rao, V.M. Defluoridation of water by activated carbon prepared from banana (Musa paradisiaca) peel and coffee (Coffe arabica) husk. Int. J. Environ. Sci. Technol. 2015, 12, 1857-1866. [CrossRef]

134. Darweesh, T.M.; Ahmed, M.J. Batch and fixed bed adsorption of levofloxacin on granular activated carbon from date (Phoenix dactylifera L.) stones by $\mathrm{KOH}$ chemical activation. Environ. Toxicol. Pharmacol. 2017, 50, 159-166. [CrossRef]

135. Khadhri, N.; Saad, M.E.K.; ben Mosbah, M.; Moussaoui, Y. Batch and continuous column adsorption of indigo carmine onto activated carbon derived from date palm petiole. J. Environ. Chem. Eng. 2019, 7, 102775. [CrossRef]

136. Laksaci, H.; Khelifi, A.; Belhamdi, B.; Trari, M. Valorization of coffee grounds into activated carbon using physic-Chemical activation by $\mathrm{KOH} / \mathrm{CO}_{2}$. J. Environ. Chem. Eng. 2017, 5, 5061-5066. [CrossRef]

137. Wang, Y.X.; Ngo, H.H.; Guo, W.S. Preparation of a specific bamboo based activated carbon and its application for ciprofloxacin removal. Sci. Total Environ. 2015, 533, 32-39. [CrossRef] [PubMed]

138. Hashemian, S.; Salari, K.; Yazdi, Z.A. Preparation of activated carbon from agricultural wastes (almond shell and orange peel) for adsorption of 2-pic from aqueous solution. J. Ind. Eng. Chem. 2014, 20, 1892-1900. [CrossRef]

139. Talat, M.; Mohan, S.; Dixit, V.; Singh, D.K.; Hasan, S.H.; Srivastava, O.N. Effective removal of fluoride from water by coconut husk activated carbon in fixed bed column: Experimental and breakthrough curves analysis. Groundwater Sustainable Dev. 2018, 7, 48-55. [CrossRef]

140. Vu, M.T.; Chao, H.-P.; Trinh, T.V.; Le, T.T.; Lin, C.-C.; Tran, H.N. Removal of ammonium from groundwater using $\mathrm{NaOH}$-treated activated carbon derived from corncob wastes: Batch and column experiments. J. Clean. Prod. 2018, 180, 560-570. [CrossRef]

141. Dhorabe, P.T.; Lataye, D.H.; Ingole, R.S. Removal of 4-nitrophenol from aqueous solution by adsorption onto activated carbon prepared from Acacia glauca sawdust. Water Sci. Technol. 2016, 73, 955-966. [CrossRef] [PubMed]

142. Zhang, Z.; Luo, X.; Liu, Y.; Zhou, P.; Ma, G.; Lei, Z.; Lei, L. A low cost and highly efficient adsorbent (activated carbon) prepared from waste potato residue. J. Taiwan Inst. Chem. Eng. 2015, 49, 206-211. [CrossRef]

143. Osman, A.I.; Blewitt, J.; Abu-Dahrieh, J.K.; Farrell, C.; Al-Muhtaseb, A.H.; Harrison, J.; Rooney, D.W. Production and characterisation of activated carbon and carbon nanotubes from potato peel waste and their application in heavy metal removal. Environ. Sci. Pollut. Res. 2019, 26, 37228-37241. [CrossRef]

144. Satayeva, A.R.; Howell, C.A.; Korobeinyk, A.V.; Jandosov, J.; Inglezakis, V.J.; Mansurov, Z.A.; Mikhalovsky, S.V. Investigation of rice husk derived activated carbon for removal of nitrate contamination from water. Sci. Total Environ. 2018, 630, 1237-1245. [CrossRef] 
145. Baysal, M.; Bilge, K.; Yilmaz, B.; Papila, M.; Yürüm, Y. Preparation of high surface area activated carbon from waste-biomass of sunflower piths: Kinetics and equilibrium studies on the dye removal. J. Environ. Chem. Eng. 2018, 6, 1702-1713. [CrossRef]

146. Fu, K.; Yue, Q.; Gao, B.; Wang, Y.; Li, Q. Activated carbon from tomato stem by chemical activation with $\mathrm{FeCl}_{2}$. Colloids Surf. A 2017, 529, 842-849. [CrossRef]

147. Liew, R.K.; Azwar, E.; Yek, P.N.Y.; Lim, X.Y.; Cheng, C.K.; Ng, J.-H.; Jusoh, A.; Lam, W.H.; Ibrahim, M.D.; $\mathrm{Ma}$, N.L.; et al. Microwave pyrolysis with $\mathrm{KOH} / \mathrm{NaOH}$ mixture activation: A new approach to produce micro-mesoporous activated carbon for textile dye adsorption. Bioresour. Technol. 2018, 266, 1-10. [CrossRef] [PubMed]

148. Ioannidou, O.; Zabaniotou, A. Agricultural residues as precursors for activated carbon production-a review. Renew. Sustain. Energy Rev. 2007, 11, 1966-2005. [CrossRef]

149. Pekala, R.W.; Kong, F.M. Proc. 2nd Int. Symp. of Aerogels (ISA2). Rev. Phys. Appl. 1989, 24 C4, 33.

150. Pekala, R.W. Organic aerogels from the polycondensation of resorcinol with formaldehyde. J. Mater. Sci. 1989, 24, 3221-3227. [CrossRef]

151. Yamamoto, Y.; Sugimoto, T.; Suzuki, T.; Mukai, S.R.; Tamon, H. Preparation and characterization of carbon cryogel microspheres. Carbon 2002, 40, 1345-1351. [CrossRef]

152. Saliger, R.; Bock, V.; Petrcevic, R.; Tillostson, T.; Geis, S.; Fricke, J. Carbon aerogels from dilute catalysis of resorcinol with formaldehyde. J. Non-Cryst. Solids 1997, 221, 144-150. [CrossRef]

153. Moussaoui, Y.; Elaloui, E.; Ben Salem, R. Etude mécanistique et structurale des résines R/F. C. R. Chimie 2012, 15, 493-498. [CrossRef]

154. Bock, V.; Emmerling, A.; Fricke, J. Influence of monomer and catalyst concentration on RF and carbon aerogel structure. J. Non-Cryst. Solids 2005, 225, 69-73. [CrossRef]

155. Wei, W.; Hu, H.; Qin, G.; You, L.; Chen, G. Pore structure control of phenol-formaldehyde based carbon microfiltration membranes. Carbon 2004, 42, 679-681. [CrossRef]

156. Tonanon, N.; Siyasukh, A.; Tanthapanichakoon, W.; Nishihara, H.; Mukai, S.R.; Tamon, H. Improvement of mesoporosity of carbon cryogels by ultrasonic irradiation. Carbon 2005, 43, 525-531. [CrossRef]

157. Moussaoui, R.; ben mosbah, M.; Moussaoui, Y.; Elaloui, E. Structural Study of Microporous Xerogels Prepared by Polycondensation of Pyrogallol with Formaldehyde. J. Adv. Chem. 2013, 6, 878-883. [CrossRef]

158. Moussaoui, R.; ben Mosbah, M.; Elghniji, K.; Elaloui, E.; Moussaoui, Y. Sol-gel synthesis of microporous carbon using Resorcinol and Formaldehyde. J. Chem. Res. 2016, 40, 209-212. [CrossRef]

159. Ma, M.; Ying, H.; Cao, F.; Wang, Q.; Ai, N. Adsorption of congo red on mesoporous activated carbon prepared by $\mathrm{CO}_{2}$ physical activation. Chin. J. Chem. Eng. 2020, 28, 1069-1076. [CrossRef]

160. Rambabu, N.; Rao, B.V.S.K.; Surisetty, V.R.; Das, U.; Dalai, A.K. Production, characterization and evaluation of activated carbons from de-oiled canola meal for environmental applications. Ind. Crop. Prod. 2015, 65, 572-581. [CrossRef]

161. Guoa, Y.; Tan, C.; Sun, J.; Li, W.; Zhang, J.; Zhao, C. Porous activated carbons derived from waste sugarcane bagasse for $\mathrm{CO}_{2}$ adsorption. Chem. Eng. J. 2020, 381, 122736. [CrossRef]

162. Pezoti, O.; Cazetta, A.L.; Bedin, K.C.; Souza, L.S.; Martins, A.C.; Silva, T.L.; Júnior, O.O.S.; Visentainer, J.V.; Almeida, V.C. NaOH-activated carbon of high surface area produced from guava seeds as a high-efficiency adsorbent for amoxicillin removal: Kinetic, isotherm and thermodynamic studies. Chem. Eng. J. 2016, 288, 778-788. [CrossRef]

163. Ibrahim, W.M.; Hassan, A.F.; Azab, Y.A. Biosorption of toxic heavy metals from aqueous solution by Ulva lactuca activated carbon. Egypt. J. Basic Appl. Sci. 2016, 3, 241-249. [CrossRef]

164. Benaddi, H.; Legras, D.; Rouzaud, J.N.; Beguin, F. Influence of the atmosphere in the chemical activation of wood by phosphoric acid. Carbon 1998, 36, 306-309. [CrossRef]

165. Heidari, A.; Younesi, H.; Rashidi, A.; Ghoreyshi, A.A. Adsorptive removal of $\mathrm{CO}_{2}$ on highly microporous activated carbons prepared from Eucalyptus camaldulensis wood: Effect of chemical activation. J. Taiwan Inst. Chem. Eng. 2014, 45, 579-588. [CrossRef]

166. Nam, H.; Wang, S.; Jeong, H.R. TMA and $\mathrm{H}_{2} \mathrm{~S}$ gas removals using metal loaded on rice husk activated carbonfor indoor air purification. Fuel 2018, 213, 186-194. [CrossRef]

167. Wang, S.; Nam, H.; Nam, H. Preparation of activated carbon from peanut shell with $\mathrm{KOH}$ activation and its application for $\mathrm{H}_{2} \mathrm{~S}$ adsorption in confined space. J. Environ. Chem. Eng. 2020, 8, 103683. [CrossRef] 
168. Demiral, H.; Güngor, C. Adsorption of copper(II) from aqueous solutions on activated carbon prepared from grape bagasse. J. Cleaner Prod. 2016, 124, 103-113. [CrossRef]

169. Gueye, M.; Richardson, Y.; Kafack, F.T.; Blin, J. High efficiency activated carbons from African biomass residues for the removal of chromium(VI) from wastewater. J. Environ. Chem. Eng. 2014, 2, $273-281$. [CrossRef]

170. Danish, M.; Hashim, R.; Ibrahim, M.N.M.; Sulaiman, O. Optimization study for preparation of activated carbon from Acacia mangium wood using phosphoric acid. Wood Sci. Technol. 2014, 48, 1069-1083. [CrossRef]

171. Girgis, B.S.; El-Hendawy, A.A. Porosity development in activated carbons obtained from date pits under chemical activation with phosphoric acid. Microporous Mesoporous Mater. 2002, 52, 105-117. [CrossRef]

172. Abdel-Ghani, N.T.; El-Chaghaby, G.A.; ElGammal, M.H.; Rawash, E.-S.A. Optimizing the preparation conditions of activated carbons from olive cake using KOH activation. New Carbon Mater. 2016, 31, 492-500. [CrossRef]

173. Mahmoudi, K.; Hamdi, N.; Srasra, E. Preparation and characterization of activated carbon from date pits by chemical activation with zinc chloride for methyl orange adsorption. J. Mater. Environ. Sci. 2014, 5, 1758-1769.

174. Wu, F.C.; Tseng, R.L.; Juang, R.S. Preparation of highly microporous carbons from fir wood by $\mathrm{KOH}$ activation for adsorption of dyes and phenols from water. Sep. Purif. Technol. 2005, 47, 10-19. [CrossRef]

175. Rahman, A.; Hango, H.J.; Daniel, L.S.; Uahengo, V.; Jaime, S.J.; Bhaskaruni, S.V.H.S.; Jonnalagadda, S.B. Chemical preparation of activated carbon from Acacia erioloba seed pods using $\mathrm{H}_{2} \mathrm{SO}_{4}$ as impregnating agent for water treatment: An environmentally benevolent approach. J. Cleaner Prod. 2019, 237, 117689. [CrossRef]

176. Naeem, S.; Baheti, V.; Wiener, J.; Marek, J. Removal of methylene blue from aqueous media using activated carbon web. J. Text. Inst. 2016, 108, 803-811. [CrossRef]

177. Marzbali, M.H.; Esmaieli, M. Fixed bed adsorption of tetracycline on a mesoporous activated carbon: Experimental study and neuro-fuzzy modeling. J. Appl. Res. Technol. 2017, 15, 454-463. [CrossRef]

178. Li, Z.; Hanafy, H.; Zhang, L.; Sellaoui, L.; Netto, M.S.; Oliveira, M.L.S.; Seliem, M.K.; Dotto, G.L.; Bonilla-Petriciolet, A.; Li, Q. Adsorption of congo red and methylene blue dyes on an ashitaba waste and a walnut shell-based activated carbon from aqueous solutions: Experiments, characterization and physical interpretations. Chem. Eng. J. 2020, 388, 124263. [CrossRef]

179. Kalra, A.; Hadi, P.; Hui, C.W.; Mackey, H.; Ansari, T.A.; Saleem, J.; McKay, G. Adsorption of Dyes from Water on to Bamboo-Based Activated Carbon-Error Analysis Method for Accurate Isotherm Parameter Determination. J. Water Sci. Eng. 2019, 1, 1-11.

180. Liu, Y.; Cheng, H.; He, Y. Application and Mechanism of Sludge-Based Activated Carbon for Phenol and Cyanide Removal from Bio-Treated Effluent of Coking Wastewater. Processes 2020, 8, 82. [CrossRef]

181. Nowicki, P.; Kazmierczak-Razna, J.; Pietrzak, R. Physicochemical and adsorption properties of carbonaceous sorbents prepared by activation of tropical fruit skins with potassium carbonate. Mater. Des. 2016, 90, 579-585. [CrossRef]

182. Nirmala, G.; Murugesan, T.; Rambabu, K.; Sathiyanarayanan, K.; Show, P.L. Adsorptive removal of phenol using banyan root activated carbon. Chem. Eng. Commun. 2019. [CrossRef]

183. Tu, B.; Wen, R.; Wang, K.; Cheng, Y.; Deng, Y.; Cao, W.; Zhang, K.; Tao, H. Efficient removal of aqueous hexavalent chromium by activated carbon derived from Bermuda grass. J. Colloid Interface Sci. 2020, 560, 649-658. [CrossRef]

184. Thabede, P.M.; Shooto, N.D.; Naidoo, E.B. Removal of methylene blue dye and lead ions from aqueous solution using activated carbon from black cumin seeds. S. Afr. J. Chem. Eng. 2020, 33, 39-50. [CrossRef]

185. Lütke, S.F.; Igansi, A.V.; Pegoraro, L.; Dotto, G.L.; Pinto, L.A.A.; Cadaval, T.R.S. Preparation of activated carbon from black wattle bark waste and its application for phenol adsorption. J. Environ. Chem. Eng. 2019, 7, 103396.

186. Kasperiski, F.M.; Lima, E.C.; Umpierres, C.S.; dos Reis, G.S.; Thue, P.S.; Lima, D.R.; Dias, S.L.P.; Saucier, C.; da Costa, J.B. Production of porous activated carbons from Caesalpinia ferrea seed pod wastes: Highly efficient removal of captopril from aqueous solutions. J. Clean. Prod. 2018, 197, 919-929. [CrossRef]

187. Aguayo-Villarreal, I.A.; Bonilla-Petriciolet, A.; Muñiz-Valencia, R. Preparation of activated carbons from pecan nutshell and their application in the antagonistic adsorption of heavy metal ions. J. Mol. Liq. 2017, 230, 686-695. [CrossRef] 
188. Dwivedi, A.D.; Dubey, S.P.; Sillanpää, M.; Kwon, Y.-N.; Lee, C. Distinct adsorption enhancement of bi-component metals (cobalt and nickel) by Fireweed-derived carbon compared to activated carbon: Incorporation of surface group distributions for increased efficiency. Chem. Eng. J. 2015, 281, 713-723. [CrossRef]

189. Islam, M.A.; Ahmed, M.J.; Khanday, W.A.; Asif, M.; Hameed, B.H. Mesoporous activated coconut shell-derived hydrochar prepared via hydrothermal carbonization-NaOH activation for methylene blue adsorption. J. Environ. Manag. 2017, 203, 237-244. [CrossRef] [PubMed]

190. Jain, A.; Balasubramanian, R.; Srinivasan, M.P. Tuning hydrochar properties for enhanced mesopore development in activated carbon by hydrothermal carbonization. Microporous Mesoporous Mater. 2015, 203, 178-185. [CrossRef]

191. Rodiguez, M.H.; Yperman, J.; Carleer, R.; Maggen, J.; Dadi, D.; Gryglewicz, G.; der Bruggen, B.V.; Hernández, J.F.; Calvis, A.O. Adsorption of $\mathrm{Ni}(\mathrm{II})$ on spent coffee and coffee husk based activated carbon. J. Environ. Chem. Eng. 2018, 6, 1161-1170. [CrossRef]

192. Liu, Z.; Sun, Y.; Xu, X.; Meng, X.; Qu, J.; Wang, Z.; Liu, C.; Qu, B. Preparation, characterization and application of activated carbon from corn cob by $\mathrm{KOH}$ activation for removal of $\mathrm{Hg}$ (II) from aqueous solution. Bioresour. Technol. 2020, 306, 123154. [CrossRef]

193. Zhang, Y.; Song, X.; Zhang, P.; Gao, H.; Ou, C.; Kong, X. Production of activated carbons from four wastes via one-step activation and their applications in $\mathrm{Pb}^{2+}$ adsorption: Insight of ash content. Chemosphere 2020, 245, 125587. [CrossRef]

194. Yu, X.; Han, X.; Chang, C.; Hu, Y.; Xu, C.C.; Fang, S. Corncob-derived activated carbon for roxarsone removal from aqueous solution: Isotherms, kinetics, and mechanism. Environ. Sci. Pollut. Res. 2020, 27, 15785-15797. [CrossRef]

195. Yazidi, A.; Atrous, M.; Soetaredjo, F.E.; Sellaoui, L.; Ismadji, S.; Erto, A.; Bonilla-Petriciolet, A.; Dotto, G.L.; Ben Lamine, A. Adsorption of amoxicillin and tetracycline on activated carbon prepared from durian shell in single and binary systems: Experimental study and modeling analysis. Chem. Eng. J. 2020, 379, 122320. [CrossRef]

196. Han, Q.; Wang, J.; Goodman, B.A.; Xie, J.; Liu, Z. High adsorption of methylene blue by activated carbon prepared from phosphoric acid treated eucalyptus residue. Powder Technol. 2020, 366, 239-248. [CrossRef]

197. Chen, C.; Mi, S.; Lao, D.; Shi, P.; Tong, Z.; Li, Z.; Hu, H. Single-step synthesis of eucalyptus sawdust magnetic activated carbon and its adsorption behavior for methylene blue. RSC Adv. 2019, 9, 22248-22262. [CrossRef]

198. Kumar, A.; Jena, H.M. Removal of methylene blue and phenol onto prepared activated carbon from Fox nutshell by chemical activation in batch and fixed-bed column. J. Clean. Prod. 2016, 137, 1246-1259. [CrossRef]

199. Kumar, A.; Jena, H.M. Adsorption of Cr(VI) from aqueous solution by prepared high surface area activated carbon from Fox nutshell by chemical activation with $\mathrm{H}_{3} \mathrm{PO}_{4}$. J. Environ. Chem. Eng. 2017, 5, 2032-2041. [CrossRef]

200. Sayğili, H.; Güzel, F.; Önal, Y. Conversion of grape industrial processing waste to activated carbon sorbent and its performance in cationic and anionic dyes adsorption. J. Clean. Prod. 2015, 93, 84-93. [CrossRef]

201. Nagalakshmi, T.V.; Emmanuel, K.A.; Bhavani, P. Adsorption of disperse blue 14 onto activated carbon prepared from Jackfruit-PPI-I waste. Mater. Today: Proc. 2019, 18, 2036-2051. [CrossRef]

202. Shokry, H.; Elkady, M.; Hamad, H. Nano activated carbon from industrial mine coal as adsorbents for removal of dye from simulated textile wastewater: Operational parameters and mechanism study. J. Mater. Res. Technol. 2019, 8, 4477-4488. [CrossRef]

203. Al-Ghouti, M.A.; Sweleh, A.O. Optimizing textile dye removal by activated carbon prepared from olive stones. Environ. Technol. Innovation 2019, 16, 100488. [CrossRef]

204. Elhleli, H.; Mannai, F.; Elaloui, E.; Moussaoui, Y. Nitrophenol Removal from Wastewater onto Prepared Activated Carbon from Opuntia ficus-indica. In Recent Advances in Environmental Science from the Euro-Mediterranean and Surrounding Regions; Kallel, A., Ksibi, M., Ben Dhia, H., Khélifi, N., Eds.; EMCEI 2017, Advances in Science, Technology \& Innovation (IEREK Interdisciplinary Series for Sustainable Development); Springer: Cham, Switzerland, 2018; pp. 1245-1246.

205. Pamidimukkala, P.S.; Soni, H. Efficient removal of organic pollutants with activated carbon derived from palm shell: Spectroscopic characterisation and experimental optimization. J. Environ. Chem. Eng. 2018, 6, 3135-3149. [CrossRef] 
206. da Paixao Cansado, I.P.; Belo, C.R.; Mourao, P.A.M. Pesticides abatement using activated carbon produced from a mixture of synthetic polymers by chemical activation with $\mathrm{KOH}$ and $\mathrm{K}_{2} \mathrm{CO}_{3}$. Environ. Nanotechnol. Monit. Manag. 2019, 12, 100261. [CrossRef]

207. Bedada, D.; Angassa, K.; Tiruneh, A.; Kloos, H.; Fito, J. Chromium removal from tannery wastewater through activated carbon produced from Parthenium hysterophorus weed. Energ. Ecol. Environ. 2020, 5, 184-195. [CrossRef]

208. Xu, W.; Zhao, Q.; Wang, R.; Jiang, Z.; Zhang, Z.; Gao, X.; Ye, Z. Optimization of organic pollutants removal from soil eluent by activated carbon derived from peanut shells using response surface methodology. Vacuum 2017, 141, 307-315. [CrossRef]

209. Danish, M.; Khanday, W.A.; Hashim, R.; Sulaiman, N.S.B.; Akhtar, M.N.; Nizami, M. Application of optimized large surface area date stone (Phoenix dactylifera) activated carbon for rhodamin B removal from aqueous solution: Box-Behnken design approach. Ecotoxicol. Environ. Saf. 2017, 139, 280-290. [CrossRef] [PubMed]

210. Darweesh, T.M.; Ahmed, M.J. Adsorption of ciprofloxacin and norfloxacin from aqueous solution onto granular activated carbon in fixed bed column. Ecotoxicol. Environ. Saf. 2017, 138, 139-145. [CrossRef] [PubMed]

211. Valizadeh, S.; Younesi, H.; Bahramifar, N. Highly mesoporous $\mathrm{K}_{2} \mathrm{CO}_{3}$ and $\mathrm{KOH} /$ activated carbon for SDBS removal from water samples: Batch and fixed-bed column adsorption process. Environ. Nanotechnol. Monit. Manag. 2016, 6, 1-13. [CrossRef]

212. Arampatzidou, A.C.; Deliyanni, E.A. Comparison of activation media and pyrolysis temperature for activated carbons development by pyrolysis of potato peels for effective adsorption of endocrine disruptor bisphenol-A. J. Colloid Interface Sci. 2016, 466, 101-112. [CrossRef]

213. Runtti, H.; Tuomikoski, S.; Kangas, T.; Lassi, U.; Kuokkanen, T.; Rämö, J. Chemically activated carbon residue from biomass gasification as a sorbent for iron(II), copper(II) and nickel(II) ions. J. Water Process Eng. 2014, 4, 12-24. [CrossRef]

214. Bjorklund, K.; Li, L.Y. Adsorption of organic stormwater pollutants onto activated carbon from sewage sludge. J. Environ. Manag. 2017, 197, 490-497. [CrossRef]

215. Araga, R.; Soni, S.; Sharma, C.S. Fluoride adsorption from aqueous solution using activated carbon obtained from $\mathrm{KOH}$-treated jamun (Syzygium cumini) seed. J. Environ. Chem. Eng. 2017, 5, 5608-5616. [CrossRef]

216. Saygili, H.; Güzel, F. High surface area mesoporous activated carbon from tomato processing solid waste by zinc chloride activation: Process optimization, characterization and dyes adsorption. J. Clean. Prod. 2016, 113, 995-1004. [CrossRef]

217. Acosta, R.; Nabarlatz, D.; Sánchez-Sánchez, A.; Jagiello, J.; Gadonneix, P.; Celzard, A.; Fierro, V. Adsorption of Bisphenol A on $\mathrm{KOH}$-activated tyre pyrolysis char. J. Environ. Chem. Eng. 2018, 6, 823-833. [CrossRef]

218. Hassan, A.F.; Elhadidy, H. Production of activated carbons from waste carpets and its application in methylene blue adsorption: Kinetic and thermodynamic studies. J. Environ. Chem. Eng. 2017, 5, 955-963. [CrossRef]

219. Nieto-Márquez, A.; Pinedo-Flores, A.; Picasso, G.; Atanes, E.; Kou, R.S. Selective adsorption of $\mathrm{Pb}^{2+}, \mathrm{Cr}^{3+}$ and $\mathrm{Cd}^{2+}$ mixtures on activated carbons prepared from waste tires. J. Environ. Chem. Eng. 2017, 5, 1060-1067. [CrossRef]

220. Xiao, W.; Garba, Z.N.; Sun, S.; Lawan, I.; Wang, L.; Lin, M.; Yuan, Z. Preparation and evaluation of an effective activated carbon from white sugar for the adsorption of rhodamine B dye. J. Clean. Prod. 2020, 253, 119989. [CrossRef]

221. Gebreegziabher, T.B.; Wang, S.; Nam, H. Adsorption of $\mathrm{H}_{2} \mathrm{~S}, \mathrm{NH}_{3}$ and TMA from indoor air using porous corncob activated carbon: Isotherm and kinetics study. J. Environ. Chem. Eng. 2019, 7, 103234. [CrossRef]

222. Taleb, F.; ben Mosbah, M.; Elaloui, E.; Moussaoui, Y. Adsorption of ibuprofen sodium salt onto Amberlite resin IRN-78: Kinetics, isotherm and thermodynamic investigations. Korean J. Chem. Eng. 2017, 34, 1141-1148. [CrossRef]

223. Saad, M.K.; Khiari, R.; Elaloui, E.; Moussaoui, Y. Adsorption of anthracene using activated carbon and Posidonia oceanica. Arabian J. Chem. 2014, 7, 109-113. [CrossRef]

224. Saad, M.K.; Mnasri, N.; Mhamdi, M.; Chafik, T.; Elaloui, E.; Moussaoui, Y. Removal of methylene blue onto mineral matrices. Desalin. Water Treat. 2015, 56, 2773-2780. [CrossRef]

225. Baheti, V.; Naeem, S.; Militky, J.; Okrasa, M.; Tomkova, B. Optimized preparation of activated carbon nanoparticles from acrylic fibrous wastes. Fibers Polym. 2015, 16, 2193-2201. [CrossRef] 
226. Fan, S.; Wang, Y.; Wang, Z.; Tang, J.; Tang, J.; Li, X. Removal of methylene blue from aqueous solution by sewage sludge-derived biochar: Adsorption kinetics, equilibrium, thermodynamics and mechanism. J. Environ. Chem. Eng. 2017, 5, 601-611. [CrossRef]

227. Abdelhafez, A.A.; Li, J. Removal of $\mathrm{Pb}$ (II) from aqueous solution by using biochars derived from sugar cane bagasse and orange peel. J. Taiwan Inst. Chem. Eng. 2016, 61, 367-375. [CrossRef]

228. Liu, L.; Li, Y.; Fan, S. Preparation of $\mathrm{KOH}$ and $\mathrm{H}_{3} \mathrm{PO}_{4}$ Modified Biochar and Its Application in Methylene Blue Removal from Aqueous Solution. Processes 2019, 7, 891. [CrossRef]

229. Inyang, M.; Dickenson, E. The potential role of biochar in the removal of organic and microbial contaminants from potable and reuse water: A review. Chemosphere 2015, 134, 232-240. [CrossRef] [PubMed]

230. Liu, L.; Fan, S.; Li, Y. Removal Behavior of Methylene Blue from Aqueous Solution by Tea Waste: Kinetics, Isotherms and Mechanism. Int. J. Approx. Res. Public Health 2018, 15, 1321. [CrossRef] [PubMed]

231. Ramesh, T.N.; Kirana, D.V.; Ashwini, A.; Manasa, T.R. Calcium hydroxide as low cost adsorbent for the effective removal of indigo carmine dye in water. J. Saudi Chem. Soc. 2017, 21, 165-171. [CrossRef]

232. Fan, S.; Tang, J.; Wang, Y.; Li, H.; Zhang, H.; Tang, J.; Wang, Z.; Li, X. Biochar prepared from co-pyrolysis of municipal sewage sludge and tea waste for the adsorption of methylene blue from aqueous solutions: Kinetics, isotherm, thermodynamic and mechanism. J. Mol. Liq. 2016, 220, 432-441. [CrossRef]

Publisher's Note: MDPI stays neutral with regard to jurisdictional claims in published maps and institutional affiliations.

(C) 2020 by the authors. Licensee MDPI, Basel, Switzerland. This article is an open access article distributed under the terms and conditions of the Creative Commons Attribution (CC BY) license (http://creativecommons.org/licenses/by/4.0/). 